\title{
Late-Stage Neuronal Progenitors in the Retina Are Radial Müller Glia That Function as Retinal Stem Cells
}

\author{
Rebecca L. Bernardos, ${ }^{1}$ Linda K. Barthel, ${ }^{2}$ Jason R. Meyers, ${ }^{2}$ and Pamela A. Raymond ${ }^{1,2}$ \\ ${ }^{1}$ Neuroscience Program and ${ }^{2}$ Department of Molecular, Cellular, and Developmental Biology, University of Michigan, Ann Arbor, Michigan 48109-1048
}

\begin{abstract}
Neuronal progenitors in the mammalian brain derive from radial glia or specialized astrocytes. In developing neural retina, radial glia-like Müller cells are generated late in neurogenesis and are not considered to be neuronal progenitors, but they do proliferate after injury and can express neuronal markers, suggesting a latent neurogenic capacity. To examine the neurogenic capacity of retinal glial cells, we used lineage tracing in transgenic zebrafish with a glial-specific promoter ( $g f a p$, for glial fibrillary acid protein) driving green fluorescent protein in differentiated Müller glia. We found that all Müller glia in the zebrafish retina express low levels of the multipotent progenitor marker Pax6 (paired box gene 6), and they proliferate at a low frequency in the intact, uninjured retina. Müller glia-derived progenitors express $\mathrm{Crx}$ (cone rod homeobox) and are late retinal progenitors that generate the rod photoreceptor lineage in the postembryonic retina. These Müller glia-derived progenitors also remain competent to produce earlier neuronal lineages, in that they respond to loss of cone photoreceptors by specifically regenerating the missing neurons. We conclude that zebrafish Müller glia function as multipotent retinal stem cells that generate retinal neurons by homeostatic and regenerative developmental mechanisms.
\end{abstract}

Key words: retinal stem cells; Müller glia; photoreceptors; regeneration; zebrafish; radial glia

\section{Introduction}

Among the most surprising recent discoveries in neuroscience is that neural stem cells in the adult mammalian brain have characteristics of differentiated astrocytes and that radial glial cells in the embryonic neocortex can generate neurons (Anthony et al., 2004; Ever and Gaiano, 2005; Götz and Barde, 2005; Gal et al., 2006; Merkle and Alvarez-Buylla, 2006). Radial glial fibers serve as guides for immature neurons migrating to the cortical plate, a supportive role that has long been recognized (Rakic, 1972). Radial glia also give rise to adult neural stem cells in the mammalian telencephalon (Doetsch, 2003; Merkle et al., 2004). These adult neural stem cells are specialized astrocytes that, like their radial glial progenitors, maintain contact with the ventricular surface and a vascular basal lamina, properties thought to be important for sustaining the specialized microenvironment of the neurogenic niches (Alvarez-Buylla and Lim, 2004). The neurogenic capacity of some radial glia depends on expression of the homeobox gene Pax6 (paired box gene 6) (Götz and Barde, 2005). Radial glia persist in the brains of adult fish and may contribute to

\footnotetext{
Received April 11, 2007; revised May 15, 2007; accepted May 17, 2007.

This work was supported by National Institutes of Health Grant R01EY004318 (P.A.R.). R.L.B. was supported by Ruth L. Kirschstein National Research Service Individual Predoctoral Fellowship Award F31 NS047909. J.R.M. was supported by a postdoctoral fellowship from Fight for Sight and Ruth L. Kirschstein National Research Service Individual Postdoctoral Fellowship Award F32 EY017251. We thank Robert Molday (University of British Columbia, Vancouver, British Columbia, Canada) and Jonathan Scholes (University College London, London, UK) for reagents. We thank Dilip Pawar for excellent technical assistance and fish husbandry.

Correspondence should be addressed to Dr. Pamela A. Raymond, Department of Molecular, Cellular, and Developmental Biology, University of Michigan, 3003 Kraus Natural Science, 803 North University Avenue, Ann Arbor, M 48109-1048. E-mail:praymond@umich.edu.

R. L. Bernardos's present address: Department of Neurobiology, University of Massachusetts Medical School, Worcester, MA 01605.

DOI:10.1523/JNEUROSCI.1624-07.2007

Copyright $\odot 2007$ Society for Neuroscience $\quad$ 0270-6474/07/277028-13\$15.00/0
}

adult neurogenesis (Zupanc and Clint, 2003; Zupanc et al., 2005; Adolf et al., 2006).

Müller cells are the radial glia of the neural retina, and they are critical for maintaining differentiated retinal structure and function in all vertebrate species (Bringmann et al., 2006). Müller glia have a neuronal guidance role in the teleost fish retina, in which rod photoreceptors are produced continuously by rod-specific progenitors that use the radial fibers of Müller glia as guides for migration into the photoreceptor layer (Raymond and Rivlin, 1987; Julian et al., 1998; Otteson et al., 2001; Raymond et al., 2006). Lineage tracing studies in the developing retinal neuroepithelium showed that a common, multipotent retinal progenitor gives rise to all neurons and Müller glia in the vertebrate retina (Turner and Cepko, 1987; Wetts and Fraser, 1988), and the gene expression profiles of Müller glia and mitotic retinal progenitor cells in developing mouse retina substantially overlap (Blackshaw et al., 2004). Several recent reports describe expression of neuronal markers in Müller glia that proliferate after retinal injury in teleost fish (Yurco and Cameron, 2005; Fausett and Goldman, 2006; Raymond et al., 2006; Fimbel et al., 2007), early postnatal chick (Fischer and Reh, 2003; Fischer, 2005), and adult rodents (Ooto et al., 2004; Das et al., 2006). However, no studies to date have examined the question of whether Müller glia might function as neurogenic radial glia in the uninjured, developing retina. Given these tantalizing hints that Müller glia might have a latent neurogenic capacity, we asked whether Müller glia function as endogenous retinal progenitor cells.

\section{Materials and Methods}

Zebrafish care and use. We maintain an outbred colony of zebrafish (Danio rerio) and rear embryos according to standard husbandry methods (Westerfield, 2000). The Use and Care of Animals in Research Com- 
mittee at the University of Michigan approved all procedures using animals. In zebrafish, embryonic stages are from fertilization to $\sim 3 \mathrm{~d}$ postfertilization (dpf), larval stages continue to $\sim 1$ month, and juvenile fish become sexually mature adults at $\sim 3$ months of age.

Transgenic zebrafish. In the $\mathrm{Tg}(g f a p: G F P) m i 2001$ and $\mathrm{Tg}(g f a p: G F-$ P)mi2002 lines described previously (Bernardos and Raymond, 2006; Raymond et al., 2006), regulatory elements in 5' upstream and intronic regions of the glial-specific gene glial fibrillary acid protein ( $g f a p)$ drive expression of cytoplasmic enhanced green fluorescent protein (EGFP). The transgenic (Tg) line $\mathrm{Tg}(g f a p: G F P) m i 2001$ is available from the Zebrafish International Resource Center (ZIRC, Eugene, OR). To create a nuclear-targeted reporter, we fused a nuclear localization signal to the $3^{\prime}$ end of the EGFP coding sequence. The nuclear localization signal (NLS) from $p A c G F P 1-N U C$ (Clontech, Mountain View, CA) was cloned by PCR using custom-designed primers (forward, $5^{\prime}$ ccgctgtacaagtccggactcagatccc $3^{\prime}$; reverse, $5^{\prime}$ gattgcggccgcttatctagatccggtg $\left.3^{\prime}\right)$ with restriction sites added onto the $5^{\prime}$ ends (BsrGI and NotI). A XhoI restriction site in the NLS was removed by changing the codon "ctc" to "ccc" in the forward primer. The NLS sequence was then cloned into $p G E M$ and digested with BsrGI and NotI, and the NLS was ligated into pEGFP1 (Clontech), creating $p E G F P 1-N U C$. The addition of the gfap regulatory elements into the $p E G F P 1-N U C$ plasmid followed the same cloning strategy used to build the gfap:GFP vector (Bernardos and Raymond, 2006). We injected this plasmid into zebrafish embryos and screened adults for germ-line incorporation of the transgene, as reported previously (Bernardos and Raymond, 2006), to create the stable transgenic line $\operatorname{Tg}(g f a p: n G F P)$ mi2004.

Retinal lesions. Ultra-high-intensity light lesions were used to produce widespread but selective loss of photoreceptors. Zebrafish ( 1 week to 2.5 months of age) were placed in $80 \mathrm{ml}$ of aquarium system water in a $100 \mathrm{ml}$ glass beaker positioned $5 \mathrm{~cm}$ from the tip of a fiber optic liquid light line (3 mm diameter) connected to an EXFO X-Cite 120W metal halide lamp (EXFO Photonic Solutions, Mississauga, Ontario, Canada). The $100 \mathrm{ml}$ beaker containing the fish was placed within a glass dish $(15 \mathrm{~cm}$ diameter) filled with water to serve as a thermal buffer. The incident light intensity on the fish was $\sim 120,000$ lux. They were illuminated for $30 \mathrm{~min}$ and then returned to aquarium system water to recover.

Bromodeoxyuridine treatment. Juvenile and adult zebrafish were placed in aquatic system water containing $5 \mathrm{~mm}$ bromodeoxyuridine (BrdU) buffered with 10 mm HEPES, pH 7.2, from 4 h up to 7 d. For long exposures, the BrdU solution was replaced every other day, and fish were fed brine shrimp on alternate days.

Immunohistochemistry. Zebrafish were killed and enucleated, and the eyes were fixed in $4 \%$ paraformaldehyde in PBS overnight at $4^{\circ} \mathrm{C}$. The tissue was cryoprotected, embedded, and sectioned as described previously (Barthel and Raymond, 1990). Cryosections, 6-8 $\mu \mathrm{m}$ thick, were incubated in $2 \mathrm{~N} \mathrm{HCl}, 0.5 \%$ Triton $\mathrm{X}-100$ in PBS for 30 min, rinsed in PBS with $0.5 \%$ Triton $\mathrm{X}-100$ for $15 \mathrm{~min}$, rinsed in PBS for $15 \mathrm{~min}$, and then processed for immunocytochemistry as described previously (Bernardos et al., 2005), except the secondary antibody incubation was increased to 2 h. For epitope retrieval (Shi et al., 1994), slides were incubated for 20 min in $10 \mathrm{~mm}$ sodium citrate and $0.05 \%$ Tween $20, \mathrm{pH} 6.0$, at $95-100^{\circ} \mathrm{C}$, allowed to cool at room temperature in the sodium citrate solution for 20 min, and then processed for immunocytochemistry. This treatment markedly enhanced the immunolabeling of nuclear proteins [Pax6, Crx (cone rod homeobox), PCNA (proliferating cell nuclear antigen), and $\mathrm{HuC}$ ].

Primary antibodies used included the following: zpr1 (1:200; ZIRC), which labels an unidentified surface epitope on double cones; Rho4D2 monoclonal antibody (1:100; from Robert Molday, University of British Columbia, Vancouver, British Columbia, Canada), generated against bovine rhodopsin; anti-HuC (mouse monoclonal generated against human $\mathrm{HuC}$ and $\mathrm{HuD}, 1: 1000$; Invitrogen, Carlsbad, CA); anti-BrdU (rat, 1:50; Accurate Chemical and Scientific Corporation, Westbury, NY); anti-Pax6 (rabbit, 1:200; Covance, Berkeley, CA); anti-Crx, a polyclonal antibody generated from a KLH-linked synthetic peptide of the C-terminal part of the zebrafish Crx protein (Shen and Raymond, 2004); anti-PCNA (mouse, 1:1000; Sigma-Aldrich, St. Louis, MO); 4C4 monoclonal antibody (1:200; from Jonathan Scholes, University College London, London, UK); and anti-GFP (rabbit, 1:500; mouse, 1:200; Invitro- gen). Secondary antibodies included the following: preabsorbed antimouse, anti-rabbit, or anti-rat cyanine 3 (Cy3), Cy5, and FITC (1:100; Jackson ImmunoResearch, West Grove, PA). The fluorescent DNAbinding dye 4',6-diamidino-2-phenylindole dihydrochloride (DAPI) (Sigma-Aldrich) was used to stain cell nuclei as described previously (Bernardos et al., 2005), and slides were coverslipped with anti-fade mounting media (60\% glycerol, $0.1 \mathrm{M}$ sodium carbonate, and $0.4 \mathrm{mg} / \mathrm{ml}$ p-phenylenediamine). For confocal microscopy, we used Prolong Gold antifade reagent with DAPI (Invitrogen).

Cell death detection. To detect cell apoptosis on light-lesioned retinal sections, we used a terminal deoxynucleotidyl transferase-mediated biotinylated UTP nick end labeling (TUNEL) kit according to the instructions of the manufacturer (Roche Applied Science, Indianapolis, IN).

Imaging. For fluorescent microscopy, we used a Zeiss AxioImager (Carl Zeiss Microimaging, Thornwood, NY) epifluorescent compound microscope equipped with an AxioCam mRM digital camera and an ApoTome for structured illumination of optical sections (also called grid projection) to generate three-dimensional optical reconstructions. Alternatively, for optical sectioning with laser scanning confocal microscopy, we used an Olympus Fluoview 500 (Olympus America, Centerville, PA) equipped with $405 \mathrm{~nm}$ blue diode, $458 \mathrm{mn}, 488 \mathrm{mn}$, and $514 \mathrm{mn}$ multiline argon, $543 \mathrm{~nm}$ helium neon green, and 633 helium neon red lasers. Images were processed (pseudocolored, $Z$-stacks converted to threedimensional projections and cut views) using Zeiss AxioVision version 4.0 or Olympus Fluoview version 4.3 with Tiempo software, respectively.

We used Adobe PhotoShop (Adobe Systems, San Jose, CA) to adjust the sharpness and levels of all images; adjustments were applied to the entire image. Black and white images were colorized red, green, blue, cyan, or magenta with the Channel mixer and Hue tools. Overlays of multiple fluorescent images from the same field of view were generated with the Layer Style tool by selecting the Screen option from the Blend Mode. Figures were constructed using Adobe Illustrator.

Statistical analysis. For quantification of changes in marker expression during regeneration, we counted cells in $100 \mu \mathrm{m}$ linear lengths of retina from $6-\mu \mathrm{m}$-thick retinal cryosections. Regions selected for analysis had to meet the following criteria: the ganglion cell layer was a single row of cells (to avoid oblique section angles) and the outer nuclear layer (ONL) showed signs of photoreceptor damage (pyknotic nuclei and/or loss of photoreceptors). The selected regions were photographed with the $40 \times$ objective. To quantify the number of GFP-positive $\left(\mathrm{GFP}^{+}\right)$nuclei in the inner nuclear layer (INL) and ONL, retinal sections from both eyes of five fish were examined at each postlesion interval $[1,2,3$, and $5 \mathrm{~d}$ postlesion (dpl)]; sections from four fish were examined at $4 \mathrm{dpl}$. For each fish, five retinal sections were counted. To quantify changes in Crx, Pax6, and PCNA expression in $\mathrm{GFP}^{+}$nuclei, five retinal sections were counted from each of three fish at each postlesion interval. One-way ANOVA and Bonferroni's post hoc test $(p<0.05)$ was used to quantify changes in expression patterns between pairs. KaleidaGraph (Synergy Software, Reading, PA) was used for statistical analysis and generation of graphs.

\section{Results}

\section{Transgenic zebrafish for lineage tracing analysis of Müller glia}

Differentiated Müller glia in teleost fish retinas endogenously express the glial-specific intermediate filament protein GFAP (Braisted et al., 1994; Julian et al., 1998; Vihtelic and Hyde, 2000; Wu et al., 2001; Yurco and Cameron, 2005). In mammals, GFAP is an astrocytic marker but is not expressed at detectable levels in Müller glia in the intact retina, although it is upregulated in response to injury in a process called reactive gliosis (Lewis and Fisher, 2003; Bringmann et al., 2006). To test the hypothesis that Müller glia are neurogenic, we used transgenic zebrafish, in which regulatory elements of the zebrafish $g$ fap gene drive expression of cytoplasmic or nuclear-targeted GFP, to lineage trace the progeny of proliferating Müller glia. In the $T g(g f a p: G F P) m i 2001$ and mi2002 transgenic lines reported previously (Bernardos and Raymond, 2006), GFP is throughout the cytoplasm of differenti- 
ated Müller glia (Fig. 1a). The cell specificity of GFP reporter expression was verified by colocalization with GFAP immunoreactivity and Müller-specific markers, including cellular retinaldehyde binding protein (CRALBP) (zebrafish gene: rlbp1like, retinaldehyde binding protein 1-like), brain lipid-binding protein (BLBP) (zebrafish gene: $f a b p-7$, for fatty acid-binding protein), and apolipoprotein E (ApoE) (zebrafish gene: $a p o E$ ) (Bernardos and Raymond, 2006; Raymond et al., 2006) (data not shown). Immunolocalization with GFAP antibody shows that intermediate filaments are concentrated in the inner radial processes, with reduced levels in the Müller cell bodies and outer radial processes. In contrast, in transgenic retinas, the cytoplasmic GFP reveals the full extent and complexity of the Müller glial processes that enwrap adjacent retinal neurons (Bernardos and Raymond, 2006).

Because the Müller glia have such extensive cell processes, to improve resolution of the lineage-tracing label and especially to allow unambiguous colocalization of GFP and neuron-specific nuclear markers, we added a nuclear localization signal to the GFP and created another stable transgenic line, $T g(g f a p: n G F P) m i 2004$. In the mi2004 line, immature Müller glia in embryos and early larval fish express the transgene, with GFP targeted to the nucleus as expected (Fig. 1b). However, as Müller glia differentiate, they downregulate expression of the $g f a p: n G F P$ transgene in the mi2004 line, for unknown reasons, and in adult fish, GFP cannot be detected in mature Müller glia in the central retina by endogenous fluorescence or by immunofluorescence with a GFP antibody. However, immature Müller glia adjacent to the ciliary marginal zone (CMZ), which is the site of continuous postembryonic retinal neurogenesis in fish (Raymond et al., 2006), transiently and specifically express the nuclear-targeted GFP, and they have a strong, endogenous fluorescent signal (Fig. 1c). When photoreceptors in the larval or adult retina are destroyed by exposure to high-intensity light (as described below), the gfap:nGFP transgene is reexpressed within $1 \mathrm{~d}$ (Fig. $1 d$ ). This is consistent with upregulation of GFAP during reactive gliosis, an injury response that occurs in Müller glia in fish (Braisted et al., 1994; Wu et al., 2001) and in mammals (Bringmann et al., 2006).

\section{Neurogenic Müller glia are $\mathrm{Pax}^{+}$and generate the rod photoreceptor lineage in the growing retina}

From previous studies, we know that Müller glia in the fish retina respond to retinal injury by reentering the cell cycle (Wu et al., 2001; Faillace et al., 2002; Yurco and Cameron, 2005; Fausett and $\boldsymbol{a}, \boldsymbol{b}, 40 \mu \mathrm{m} ; \boldsymbol{c}, \boldsymbol{d}, 30 \mu \mathrm{m}$
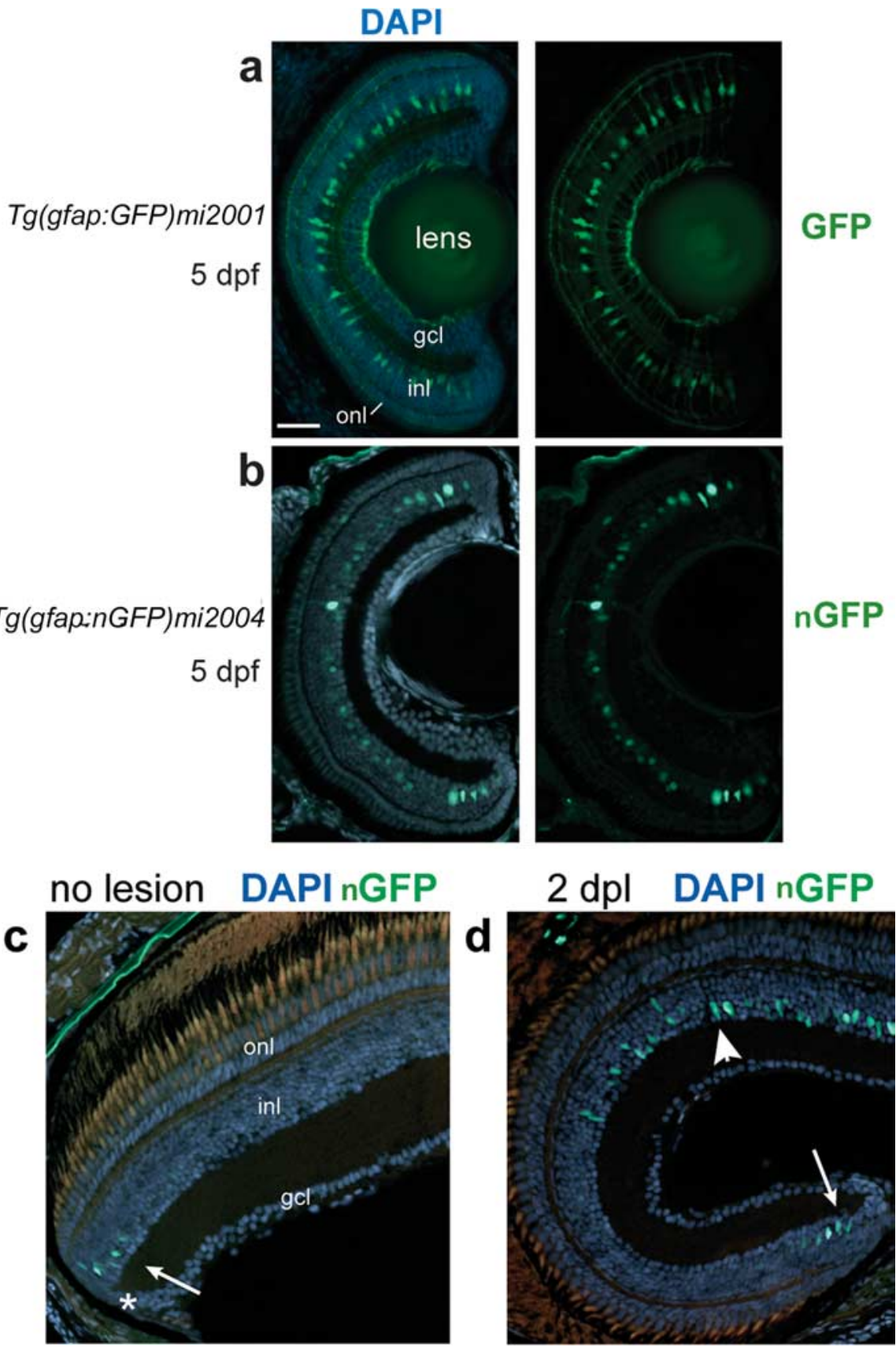

Figure 1. GFP ${ }^{+}$Müller glia in Tg(gfap:GFP)mi2001 and Tg(gfap:nGFP)mi2004 transgenic zebrafish. $\boldsymbol{a}$ - $\boldsymbol{d}$, Retinal cryosections from larval $(\boldsymbol{a}, \boldsymbol{b})$ or adult $(\boldsymbol{c}, \boldsymbol{d})$ transgenic zebrafish counterstained with DAPI (blue). $\boldsymbol{a}$, In the Tg(gfap:GFP)mi2001and mi2002 cytoplasmic GFP lines, Müller glia cell bodies in the inner nuclear layer (inl) extend radial processes toward the outer nuclear layer (onl) and ganglion cell layer ( $\mathrm{gcl}$ ). $\boldsymbol{b}$, In the Tg(gfap:nGFP)mi2004 line, reporter expression is restricted to nuclei of Müller glia in this young larval fish, at $5 \mathrm{dpf}$. The GFP expression in this preparation is visualized by immunofluorescence with an anti-GFP antibody. c, In adult Tg(gfap:nGFP)mi2004 fish, endogenous nuclear-targeted GFP expression is detected only in immature Müller glia nuclei (arrow) near the CMZ (*).d, In Tg(gfap:nGFP)mi2004 fish at $2 \mathrm{~d}$ after destruction of photoreceptors by exposure to high-intensity light (days postlesion), endogenous nuclear-targeted GFP expression is upregulated in Müller glia within the lesioned area (arrowhead) similar to immature Müller glia in newly generated retina at the ciliary margin (arrow). Scale bar: (in $\boldsymbol{a}$ )

Goldman, 2006; Vihtelic et al., 2006; Fimbel et al., 2007), but mitotic activity of Müller glia in normal, uninjured retinas has not been reported. Given the radial glial origin of neuronal progenitors during embryonic development in the mammalian cortex and the close association of progenitors in the rod lineage with radial Müller glial processes in larval (Raymond and Rivlin, 1987) and adult (Julian et al., 1998; Otteson and Hitchcock, 2003) te- 


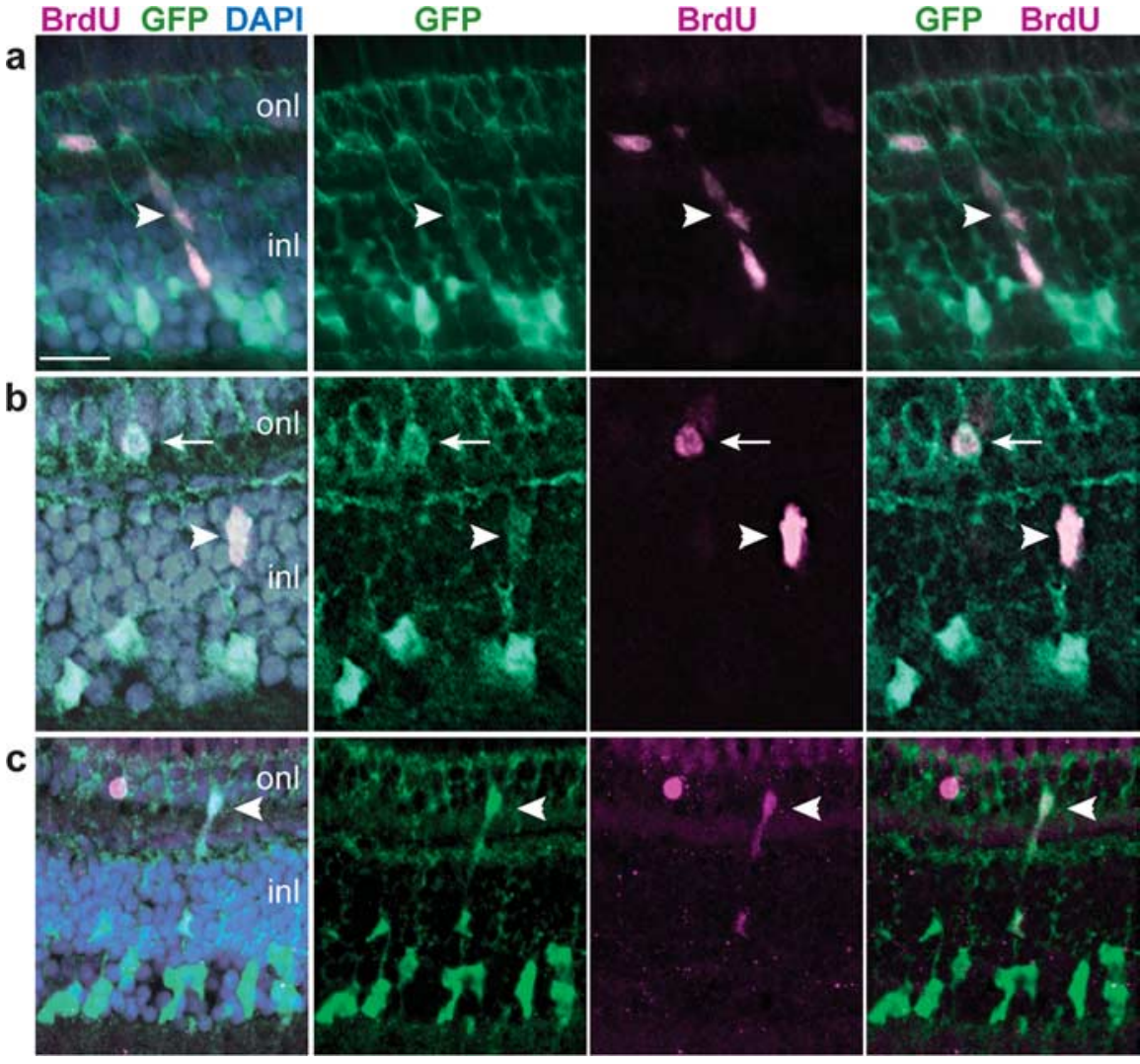

Figure 2. $\mathrm{GFP}^{+}$Müller glia proliferate in the differentiated, growing retina. $\boldsymbol{a}$ - $\boldsymbol{c}$, Retinal cryosections from juvenile $\mathrm{Tg}$ (gfap: GFP)mi2001 zebrafish labeled with BrdU (magenta) and the nuclear stain DAPI (blue). Representative images from two independent experiments, six fish per experiment, $7 \mathrm{~d}$ continuous exposure to BrdU. $\boldsymbol{a}$, A radial chain of GFP ${ }^{+}$, , BrdU $^{+}$cells (arrowhead) extends through the inner nuclear layer (inl) into the outer nuclear layer (onl). $\boldsymbol{b}, \mathrm{A}$ single GFP ${ }^{+}, \mathrm{BrdU}^{+}$cell in the INL (arrowhead) near a GFP ${ }^{+}$, , BrdU $^{+}$cell in the ONL (arrow). c, Another example of a chain of GFP ${ }^{+}$, BrdU ${ }^{+}$cells that spans INL and ONL (arrowhead). Scale bar: (in $\boldsymbol{a}) \boldsymbol{a}, \boldsymbol{b}, 15 \mu \mathrm{m} ; \boldsymbol{c}, 20 \mu \mathrm{m}$.

leost fish, we first tested the hypothesis that the Pax6 ${ }^{+}$progenitors in the INL that give rise to the rod lineage might derive from proliferating Müller glia.

To examine the kinetics of rod photoreceptor genesis, we immersed larval and juvenile $\mathrm{Tg}(g f a p$ :GFP) mi2001 zebrafish (ages from 1 week to 2.5 months old) in a HEPES-buffered solution of BrdU for $7 \mathrm{~d}$ and then examined their retinas for evidence of BrdU incorporation and rod photoreceptor genesis. We found the greatest number of $\mathrm{BrdU}^{+}$rod progenitor cells in 1- to 2-month-old juvenile fish (data not shown), so we used fish of this age in the following experiments.

To identify proliferating Müller glia, we used either PNCA immunoreactivity or BrdU incorporation (7 d continuous exposure) and looked for colocalization with GFP in transgenic fish. As expected, we found many $\mathrm{PCNA}^{+}$or $\mathrm{BrdU}^{+}$retinal progenitors in the germinal zone in the $\mathrm{CMZ}$ at the retinal periphery (data not shown). We also found evidence of rod photoreceptor genesis similar to that described previously in other teleost fish: chains of $\mathrm{PNCA}^{+}$or $\mathrm{BrdU}^{+}$cells that stretched across the INL and crossed into the ONL and were associated with Müller glia fibers (Fig. $2 a$ ). In other cases, single $\mathrm{BrdU}^{+}$cells in the INL were near one or more $\mathrm{BrdU}^{+}$cells in the ONL (Fig. 2b,c). By optical section reconstruction, we found that most of the $\mathrm{BrdU}^{+}$cells in the INL and some of those in the ONL were $\mathrm{GFP}^{+}$and therefore Müller glia or derived from Müller glia (Fig. 3a). Differentiated Müller glia are evenly distributed across the retina as seen by the regular spacing of their radial fibers (Fig. 1a). The linear density of $\mathrm{GFP}^{+}$Müller glia in the central retina of juvenile zebrafish at 1.5 months of age is $14.2 \pm 2.3$ cells per $100 \mu \mathrm{m}$ linear length in radial sections (mean $\pm \mathrm{SD} ; n=26$ ), of which $\sim 4 \%(0.6 \pm 0.4$ cells per $100 \mu \mathrm{m}$ linear length; $n=12$ ) are $\mathrm{PCNA}^{+}$and therefore actively dividing. These data show that a small fraction of differentiated Müller glia in the zebrafish retina are mitotically active and some of their progeny migrate from the INL into the ONL, forming radial chains of proliferating cells.

To demonstrate that $\mathrm{GFP}^{+}$Müllerderived cells represent progenitors in the rod lineage, we looked for coexpression of BrdU immunoreactivity with retinal progenitor markers Pax6 and Crx (Raymond et al., 2006) in nuclei of $\mathrm{GFP}^{+}$cells. In goldfish retina, $\mathrm{Pax} 6^{+}$proliferating cells in the INL have been described as putative retinal stem cells that give rise to the rod lineage (Otteson et al., 2001). We found that, in zebrafish retina, Pax6 immunoreactivity colocalized with both GFP and BrdU in the INL (Figs. $3 b-f, 4 a, b$ ), consistent with the suggestion that neuronal progenitors arise from Müller glia. The $\mathrm{GFP}^{+}, \mathrm{Pax}^{+}$nuclei did not colabel with the neuronal marker $\mathrm{HuC}$, although a separate subset of the $\mathrm{Pax}^{+}{ }^{+}$population was also $\mathrm{HuC}^{+}$(Fig. 3b); these were presumably amacrine cells. With optimal staining conditions (i.e., when slides were treated for epitope retrieval of nuclear antigens with hot sodium citrate or $2 \mathrm{~N} \mathrm{HCl}$ ), the nuclei of all $\mathrm{GFP}^{+}$Müller glia in larval, juvenile, and adult $\operatorname{Tg}(g f a p: G F P) m i 2002$ zebrafish were weakly immunoreactive with the Pax6 antibody (Fig. $3 c-f$ ). To our knowledge, the expression of Pax6 in differentiated Müller glia has not been reported previously in fish or any other vertebrate retina.

In embryonic zebrafish retina, Crx protein is expressed in late-stage neuronal progenitors, including cone and rod photoreceptor progenitors (Shen and Raymond, 2004). In the differentiated zebrafish retina, Crx expression continues in photoreceptors in the ONL as well as in a subset of neurons (probable bipolar cells) in the outer part of the INL (Shen and Raymond, 2004). In our lineage-tracing analysis of juvenile zebrafish, we found that some $\mathrm{GFP}^{+}, \mathrm{BrdU}^{+}$cells in the ONL (Fig. 4c) and occasionally in the INL (Fig. $4 d$ ) were immunoreactive for Crx. We sometimes observed pairs of $\mathrm{BrdU}^{+}$nuclei in the INL in which one was strongly $\mathrm{GFP}^{+}$and had the characteristic polygonal cell body of a Müller cell, and the other was weakly $\mathrm{Crx}^{+}$, weakly $\mathrm{GFP}^{+}$and ovoid in shape (Fig. $4 d$ ). This is consistent with an asymmetric, self-renewal division that produces a neuronal progenitor and preserves the original neurogenic Müller glia.

Finally, to confirm that $\mathrm{Pax}^{+}, \mathrm{GFP}^{+}$and $\mathrm{Crx}^{+}, \mathrm{GFP}^{+}$cells derived from proliferating Müller glia differentiate into rod photoreceptors, we used a rhodopsin antibody, Rho4D2 (Laird and Molday, 1988). We found several examples of cells that were $\mathrm{GFP}^{+}$and colabeled with both BrdU and Rho4D2 after $7 \mathrm{~d}$ exposure to BrdU (Fig. 4e). Together, these data show that proliferating Müller glia generate neuronal progenitors of the rod lin- 

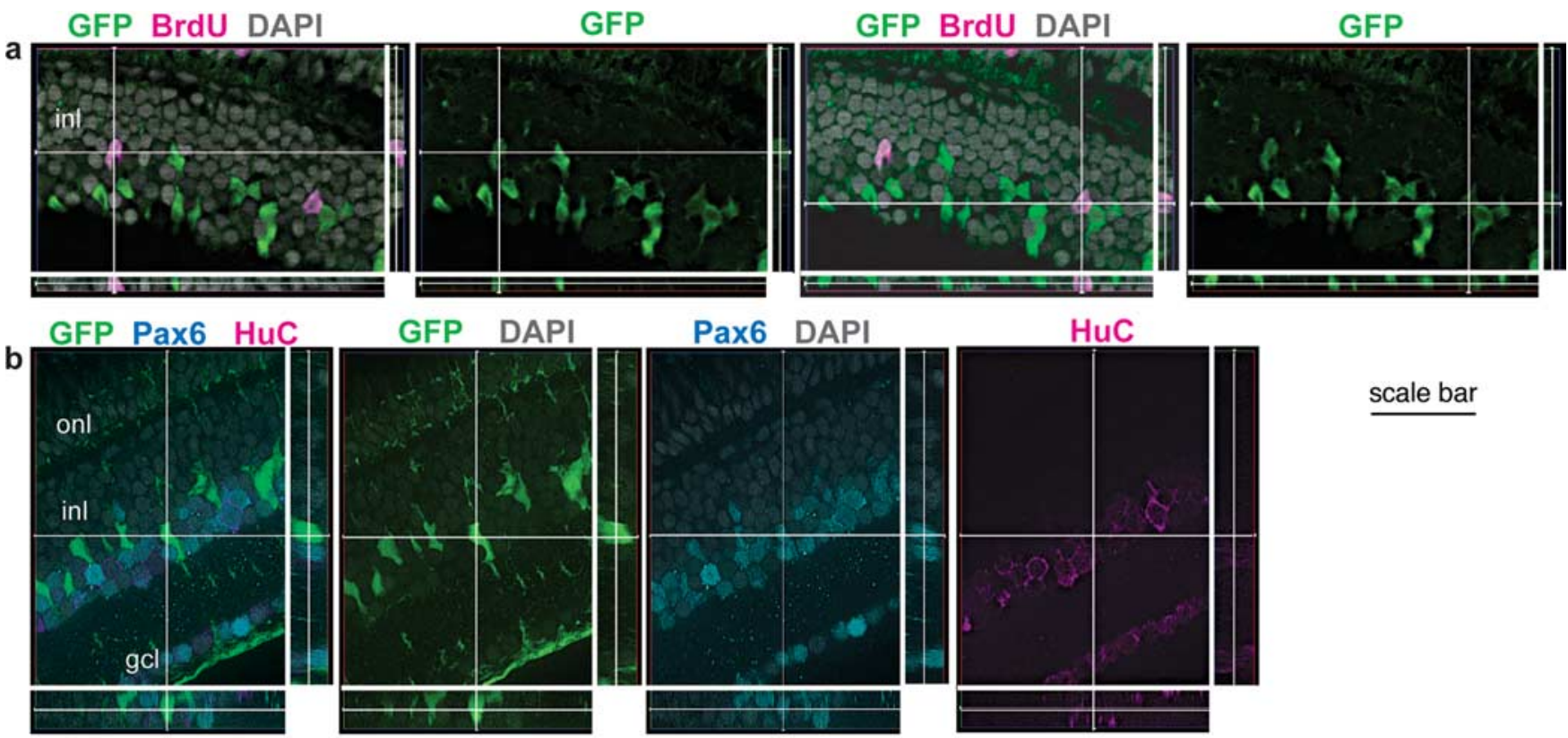

scale bar
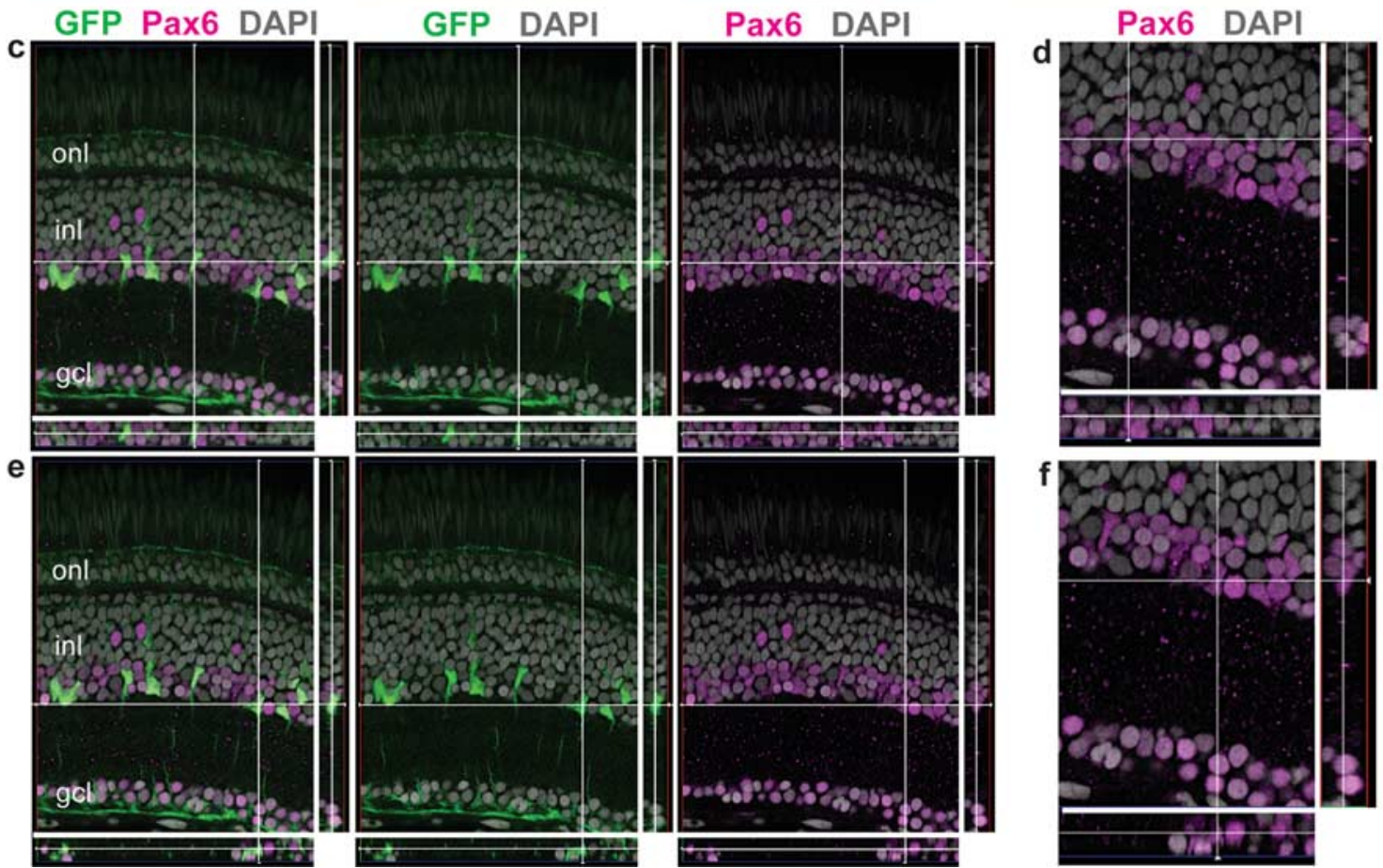

Figure 3. GFP ${ }^{+}$Müller glia are colabeled with BrdU and Pax6 but not HuC. Retinal cryosections from juvenile Tg(gfap:GFP)miz001 (a) and miz2002 (b-e) transgenic zebrafish. Reconstruction of optical slices (Z-stack) reconstructed and displayed in "cut view" through the thickness of the section in orthogonal planes. Sections were counterstained with DAPI (gray). $\boldsymbol{a}$, This fish was exposed to BrdU for $7 \mathrm{~d}$, and both of the BrdU ${ }^{+}$nuclei (magenta) in the inner nuclear layer (inl) of this image are weakly GFP ${ }^{+}$(green). $\boldsymbol{b}$, This GFP ${ }^{+}$cell (at the intersection of the crosshairs) is weakly immunoreactive for Pax6 (cyan) but not the neuronal marker HuC (magenta). As expected, many of the Pax6 ${ }^{+}$, GFP ${ }^{-}$neurons (amacrine cells in the INL) and retinal ganglion cells in the ganglion cell layer (gcl) are double labeled with HuC. $\mathbf{c - f}$, The crosshairs show two GFP ${ }^{+}$(green) Müller glia with characteristic polygonal nuclei that express Pax6, shown at higher magnification in $\boldsymbol{d}$ and $\boldsymbol{f}$, respectively. Scale bar: $\boldsymbol{a}, \boldsymbol{b}, 30 \mu \mathrm{m} ; \boldsymbol{c}, \boldsymbol{e}, 40 \mu \mathrm{m} ; \boldsymbol{d}, \boldsymbol{f}, 27 \mu \mathrm{m}$. onl, Outer nuclear layer.

eage that produce new rod photoreceptors in the differentiated, growing zebrafish retina.

Müller glia proliferate and their nuclei migrate apically after selective destruction of photoreceptors

Several previous studies of injury-induced retinal regeneration in goldfish and zebrafish suggested that Müller glia dedifferentiate, proliferate, and express neuronal markers, providing strong suggestive evidence that they may be neurogenic (Wu et al., 2001;
Yurco and Cameron, 2005; Fausett and Goldman, 2006; Raymond et al., 2006; Fimbel et al., 2007). However, none of these previous studies used a glial-specific marker for lineage tracing to show directly that the progeny of dividing Müller glia differentiate as retinal neurons. For this analysis, we developed a novel light-lesion paradigm to selectively destroy cone (and rod) photoreceptors in zebrafish (see Materials and Methods), and we specifically examined cone photoreceptor regeneration. Unlike rods, cone photoreceptors are never generated in central, differ- 

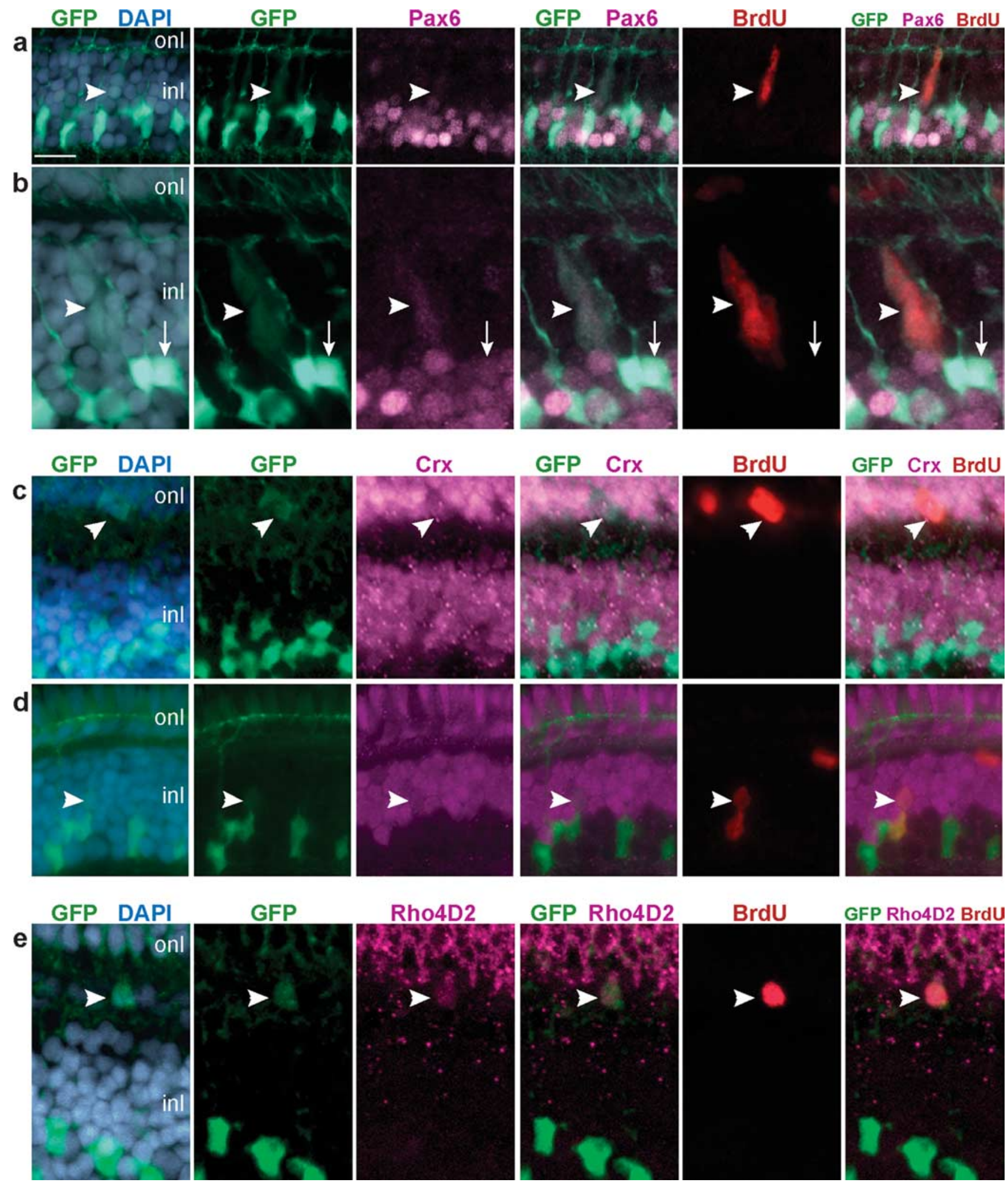

Figure 4. GFP ${ }^{+}$progenitors express neuronal and photoreceptor markers. $a-e$, Retinal cryosections from juvenile $\operatorname{Tg}(g f a p: G F P) m i z 001$ zebrafish labeled with $7 \mathrm{~d}$ continuous exposure to BrdU (red). Counterstained for nuclei with DAPI (blue) and Pax6, Crx, or Rho4D2 (magenta). $\boldsymbol{a}, \boldsymbol{b}, \mathrm{BrdU}^{+}, \mathrm{Pax}^{+}$neural progenitors in the inner nuclear layer (inl; arrowheads) are weakly labeled with GFP (green). $\boldsymbol{b}$, Nonproliferating (BrdU ${ }^{-}$) Müller glia are strongly labeled with GFP and weakly immunoreactive for Pax6 (arrow). $\boldsymbol{c}, \mathrm{A} \mathrm{GFP}^{+}, \mathrm{BrdU}^{+}$neural progenitor in the outer nuclear layer (onl) is immunoreactive for Crx (arrowhead). $\boldsymbol{d}$, A pair of BrdU ${ }^{+}$nuclei (arrowhead) in which the upper cell is $\mathrm{Crx}^{+}$and weakly $\mathrm{GFP}^{+}$, and the lower cell is $\mathrm{Crx}^{-}$, strongly GFP ${ }^{+}$, and has a polygonal-shaped nucleus characteristic of Müller glia. $\boldsymbol{e}, \mathrm{A} \mathrm{GFP}^{+}{ }^{,}, \mathrm{BrdU}^{+}$cell in the ONL is immunoreactive for rod opsin (Rho4D2; arrowhead). Scale bar: (in $\left.\boldsymbol{a}\right) \boldsymbol{a}, \boldsymbol{c}, 15 \mu \mathrm{m} ; \boldsymbol{b}, \boldsymbol{e}, 10 \mu \mathrm{m} ; \boldsymbol{d}, 12 \mu \mathrm{m}$. 
entiated regions of the intact retina (Raymond and Hitchcock, 2000; Hitchcock and Raymond, 2004). During a $30 \mathrm{~min}$ exposure to intense light, the fish became darkly pigmented as a result of melanin dispersal within melanophores in the skin. This neuroendocrine-controlled camouflage response is a response to ambient, background light levels (Muto et al., 2005), and the darkening response suggests that the intense light blinded the fish. Histological examination of the retina at 3 dpl revealed that both cone and rod photoreceptors in up to two-thirds of the central retina had degenerated (Fig. $5 a$ ), with variable photoreceptor cell loss in more peripheral regions. Cell death was specific to rods and cones in the ONL, as confirmed by TUNEL (Fig. $5 b-d$ ). The greatest number of TUNEL-labeled cells was observed at $1 \mathrm{dpl}$ (Fig. 5b), and their numbers decreased over the next few days (Fig. $5 c, d)$ as the photoreceptor debris was removed by phagocytic microglia (Fig. 5e).

By 1 month after the light lesion, cone and rod photoreceptors had regenerated and the cytoarchitecture of the retina was fully repaired (Fig. $5 g$ ). The only evidence that the photoreceptors had been destroyed and subsequently regenerated is that many photoreceptor nuclei retain the BrdU label after continuous exposure to BrdU for $72 \mathrm{~h}$, from 2 to $4 \mathrm{dpl}$, during the period of photoreceptor genesis (Raymond et al., 2006). For example, in the retina from a $T g(g f a p: G F P) m i 2002$ fish at 33 $\mathrm{dpl}$, illustrated in Figure $5 g$, many zpr $1^{+}$ cone photoreceptors are also $\mathrm{BrdU}^{+}$, and many rod nuclei in the ONL are also $\mathrm{BrdU}^{+}$.

For most of the following regeneration experiments, we used adult zebrafish from the transgenic line $\operatorname{Tg}(g f a p: n G F P) m i 2004$. The nuclear-targeted GFP provides a more discrete cell-specific marker for Müller glia and their progeny in the injured, regenerating retina compared with the cytoplasmic GFP reporter or any of a variety of Müllerspecific antibody markers that are typically used, such as glutamine synthetase, GFAP, or CRALBP (Fischer and Reh, 2003; Ooto al., 2004; Yurco and Cameron, 2005). The transgene in the mi2004 line is upregulated locally in reactive Müller glia within 1 dpl (Fig. 1d). We first quantified the proliferative response of Müller glia in lesioned retinas. The nuclei of Müller glia are normally located within one to two cell diameters of the inner boundary of the INL (Fig. $5 f$ ). At $1 \mathrm{dpl}$, the linear density of GFP ${ }^{+}$ Müller glia nuclei in the INL of retinas from light-damaged adult $\mathrm{Tg}$ (gfap: $n$ GFP) mi2004 fish was $10.5 \pm 1.8$ cells per $100 \mu \mathrm{m}$ linear length in radial sections, not significantly different from the density in uninjured retinas from adult $\operatorname{Tg}(g f a p: n G F P) m i 2001$ fish: $11.1 \pm 1.5$ cells per $100 \mu \mathrm{m}(p=1.00$, one-way ANOVA with Bonferroni's all pairs comparison post hoc test). [Note that the linear density of Müller glia in adult zebrafish is lower than the value of $14.2 \pm 0.45$ cells per $100 \mu \mathrm{m}$ in 1.5 -month-old juveniles given above; this decrease in Müller cell density in older fish reflects stretching of the retina during growth (Raymond, 1985).] However, most Müller glial nuclei within the region of the lesion had migrated apically toward the ONL (Fig. $6 a, b$ ). The nuclear migration response was strictly confined to the Müller glia whose apical processes were adjacent to damaged photoreceptors, suggesting that a local, perhaps contact-mediated, injury signal rather than a diffusible signal mediates the response. At $2 \mathrm{dpl}$, the number of $\mathrm{GFP}^{+}$nuclei in the INL increased significantly to $18.9 \pm 5.3$ per $100 \mu \mathrm{m}$ (Fig. $6 c, g)(p=0.004)$, consistent with injury-induced proliferation of Müller glia. At $3 \mathrm{dpl}$, the number of $\mathrm{GFP}^{+}$nuclei in the INL was approximately threefold that in unlesioned retinas, and this level was sustained up to $5 \mathrm{dpl}$ (Fig. $6 d, g)(p<0.0001)$. With a delay of $1-2 \mathrm{~d}, \mathrm{GFP}^{+}$nuclei also appeared in the ONL (Fig. $6 c$ ). The number of GFP ${ }^{+}$nuclei in the ONL reached $24.9 \pm 8.7$ per $100 \mu \mathrm{m}$ at $4 \mathrm{dpl}$ and then began to decline (Fig. $6 e-g$ ).

By 1 month after lesion, rod and cone photoreceptors had 

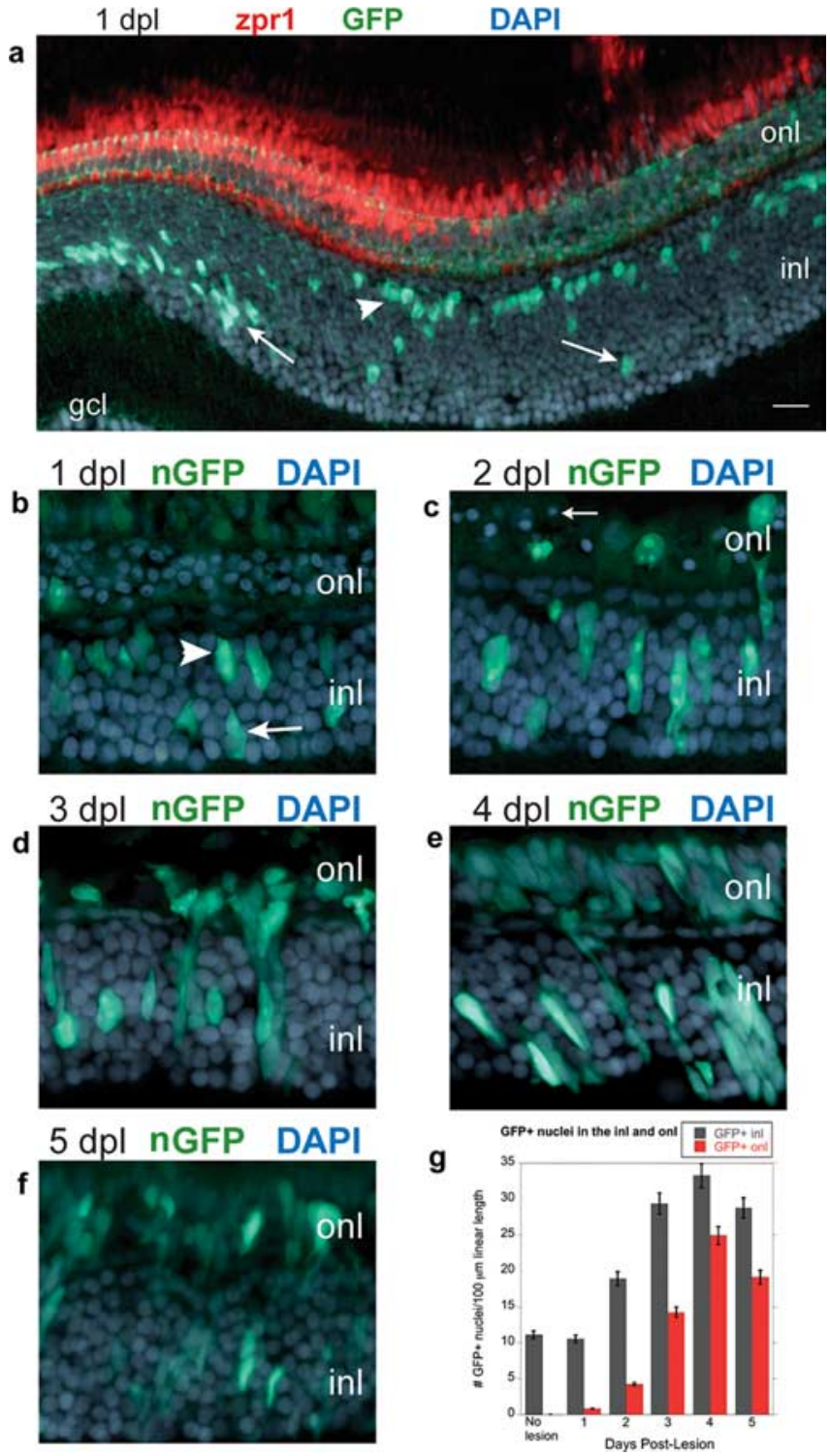

Figure 6. Müller glia proliferate after photoreceptor loss. Retinal cryosections from adult Tg(gfap:GFP)mi2001 or Tg(gfap:nGFP)mi2004 zebrafish exposed for 30 min to intense light. Sections are counterstained with DAPI (blue). a, Light-lesioned retinas from $\mathrm{Tg}$ (gfap:GFP)mi2001 zebrafish at $1 \mathrm{dpl}$ immunolabeled with the double-cone-specific zpr1 monoclonal antibody (red). Most of the GFP ${ }^{+}$Müller glia nuclei (green) within the region of the lesion have migrated from their normal position in the inner nuclear layer (inl; arrows) to the outer portion of the INL (arrowhead). $\boldsymbol{b}$-f, Light-lesioned retinas from Tg(gfap:nGFP)mi2004 zebrafish immunolabeled with anti-GFP (green). At $1 \mathrm{dpl}(\boldsymbol{b})$ and $2 \mathrm{dpl}(\boldsymbol{c}), \mathrm{GFP}^{+}$Müller nuclei are scattered throughout the INL, some have elongated, and a few have crossed into the outer nuclear layer (onl). Pyknotic photoreceptor nuclei are in the ONL (arrow). $\boldsymbol{d}$, Radially elongated clusters of $\mathrm{GFP}^{+}$nuclei are in the INL and ONL at $3 \mathrm{dpl}$ and at $4 \mathrm{dpl}(\boldsymbol{e})$. $\boldsymbol{f}$, At $5 \mathrm{dpl}$, the number of GFP ${ }^{+}$ nuclei begins to decrease in the INL and ONL. $g$, The number of GFP ${ }^{+}$nuclei in the INL (gray) and ONL (red) per $100 \mu \mathrm{m}$ linear length of retina is plotted as a function of days postlesion. Scale bar: (in $\boldsymbol{a}) \boldsymbol{a}, 20 \mu \mathrm{m} ; \boldsymbol{c}-\boldsymbol{e}, 15 \mu \mathrm{m}$. gcl, ganglion cell layer.

regenerated (Fig. $5 g$ ), and the linear density of Müller glia in the INL regenerated retina had fallen to $9.8 \pm 1.75$ cells per $100 \mu \mathrm{m}$ ( $n=19$ sections), slightly less than in unlesioned retina $(11.1 \pm$ 1.5 cells per $100 \mu \mathrm{m} ; n=16$ sections; $p<0.05$, Student's unpaired $t$ test). For these counts of Müller glia in unlesioned and regenerated (restored) retina, we used the $\mathrm{Tg}$ (gfap:GFP)mi2001 line because the GFP transgene expression is not maintained in differentiated Müller glia in the nuclear-targeted mi2004 line (Fig. 1c). In the repaired retina, both the morphology and loca- tion of Müller glial nuclei were indistinguishable from unlesioned retina (Fig. 5, compare $f, g$ ). These data demonstrate that the injury-induced, proliferating Müller glia did not generate extra glial cells, and the restoration of their normal density and morphology in the repaired retina implies that tight homeostatic control mechanisms regulate proliferation in the Müller cell compartment.

\section{$\mathrm{GFP}^{+}$cells derived from proliferating Müller glia sequentially express markers of retinal and photoreceptor progenitors}

We next asked whether any of the $\mathrm{GFP}^{+}$progeny of Müller glia were photoreceptor progenitors that differentiated into cone and rod photoreceptors to replace those destroyed by the light lesion. We looked for coexpression of GFP with PCNA and Pax6 or Crx at $1-5 \mathrm{dpl}$. For these experiments, we used the $\mathrm{Tg}$ (gfap:nGFP)mi2004 line, which greatly enhanced our ability to trace the fate of the $\mathrm{GFP}^{+}$progeny because all of these markers are nuclear signals and colocalize with the nuclear GFP immunofluorescence.

In the unlesioned retina, Pax6 immunoreactivity in the INL is primarily restricted to nuclei in the inner half (Fig. 7c). At 1 and 2 $\mathrm{dpl}$ after light lesions, we found rare $\mathrm{GFP}^{+}, \mathrm{PCNA}^{+}$nuclei in the INL that were weakly Pax6 immunoreactive $(0.67 \pm 1.6$ and $0.33 \pm 0.49$ nuclei per $100 \mu \mathrm{m}$, respectively) (Fig. $7 d$ ) but none in the ONL at these postlesion intervals. At $3 \mathrm{dpl}$ (Fig. 7a) and $4 \mathrm{dpl}$ (Fig. $7 b$ ), regularly spaced, radially oriented clusters of PCNA ${ }^{+}$, $\mathrm{Pax}^{+}{ }^{+}$cells were associated with each Müller glial cell. These neurogenic clusters are well known hallmarks of retinal regeneration in teleost fish (Raymond et al., 1988; Vihtelic and Hyde, 2000; Faillace et al., 2002; Yurco and Cameron, 2005). The population of $\mathrm{GFP}^{+}, \mathrm{PCNA}^{+}, \mathrm{Pax}^{+}$nuclei in the INL peaked at 3 and $4 \mathrm{dpl}(14.5 \pm 5.0$ nuclei per $100 \mu \mathrm{m})$ and then decreased sharply to $6.0 \pm 4.1$ nuclei per $100 \mu \mathrm{m}$ at $5 \mathrm{dpl}$ (Fig. $7 b, d)(p<$ $0.0001)$. In contrast, only a few $\mathrm{GFP}^{+}, \mathrm{PCNA}^{+}, \mathrm{Pax}^{+}$nuclei were in the ONL at 3 and $4 \mathrm{dpl}(4.3 \pm 4.6$ and $5.1 \pm 5.1$ nuclei per $100 \mu \mathrm{m}$, respectively) (Fig. $7 a, b, d$ ) and almost none at $5 \mathrm{dpl}$ $(0.93 \pm 1.5$ nuclei per $100 \mu \mathrm{m})$. We rarely found $\mathrm{PCNA}^{+}, \mathrm{Pax}^{+}$ neuronal progenitors that lacked GFP and therefore might not have been derived from proliferating Müller glia: in a total of 75 retinal sections examined (from three different fish), only six $\mathrm{Pax}^{+}, \mathrm{PCNA}^{+}$nuclei in the INL were GFP negative $\left(\mathrm{GFP}^{-}\right)$ (three at $1 \mathrm{dpl}$; one at $2 \mathrm{dpl}$; two at $4 \mathrm{dpl}$ ).

We next looked for evidence of photoreceptor specification within the $\mathrm{GFP}^{+}$lineage in regenerating retinas. In the unlesioned retina, Crx immunoreactivity is found in nuclei of both cone and rod photoreceptors in the ONL and nuclei in the outer half of the INL (Fig. 8c). At 2 and $3 \mathrm{dpl}$ after light lesions, we found occasional $\mathrm{GFP}^{+}, \mathrm{PCNA}^{+}$nuclei that colabeled with the photoreceptor progenitor marker $\operatorname{Crx}(0.27 \pm 0.59$ and $3.3 \pm 2.6$ nuclei per $100 \mu \mathrm{m}$, respectively). At $3 \mathrm{dpl}, \mathrm{GFP}^{+}, \mathrm{PCNA}^{+}, \mathrm{Crx}^{+}$ nuclei were also ONL $(2.9 \pm 2.5$ nuclei per $100 \mu \mathrm{m})$ (Fig. $8 a)$, and they increased significantly in number at 4 and $5 \mathrm{dpl}(14.9 \pm 8.7$ and $13.1 \pm 4.4$ nuclei per $100 \mu \mathrm{m}$, respectively; $p<0.0001$ compared with $3 \mathrm{dpl}$ ) (Fig. 8b,d). At $4 \mathrm{dpl} \mathrm{GFP}{ }^{+}, \mathrm{PCNA}^{+}, \mathrm{Crx}^{+}$ nuclei were approximately threefold more abundant in the ONL compared with the INL $(4.6 \pm 4.0$ nuclei per $100 \mu \mathrm{m} ; p<$ 0.0005). Because the Pax6 and Crx antibodies are both rabbit polyclonals, we could not use double immunolabeling, but the spatiotemporal distribution of $\mathrm{Pax}^{+}{ }^{+}$and $\mathrm{Crx}^{+}$immunolabeled $\mathrm{GFP}^{+}, \mathrm{BrdU}^{+}$cells is consistent with a transition from Pax6 to Crx expression in progenitors as they migrate from INL to ONL.

Together, the time course and distribution of the GFP lineage tracer and expression of the transcription factors Pax6 and Crx 

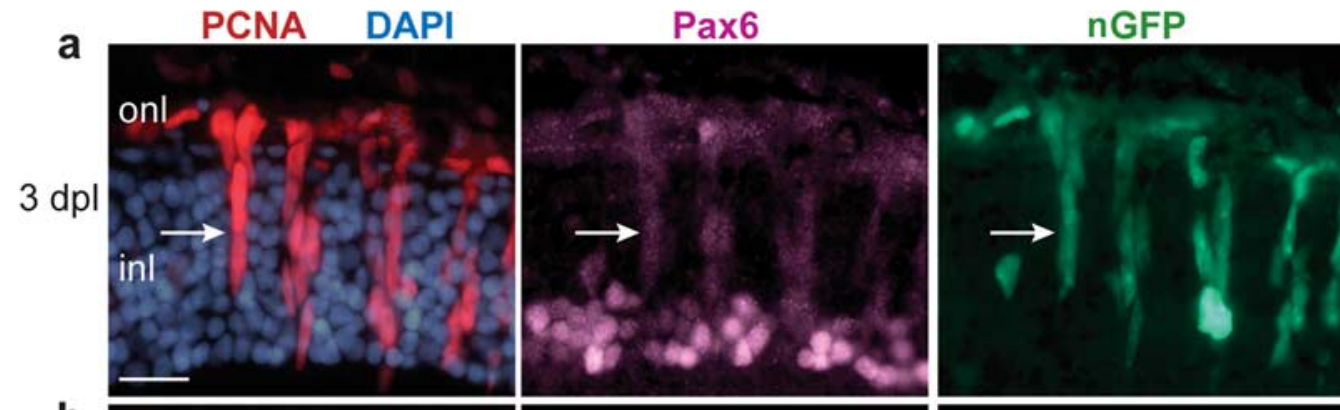

Pax6 PCNA nGFP
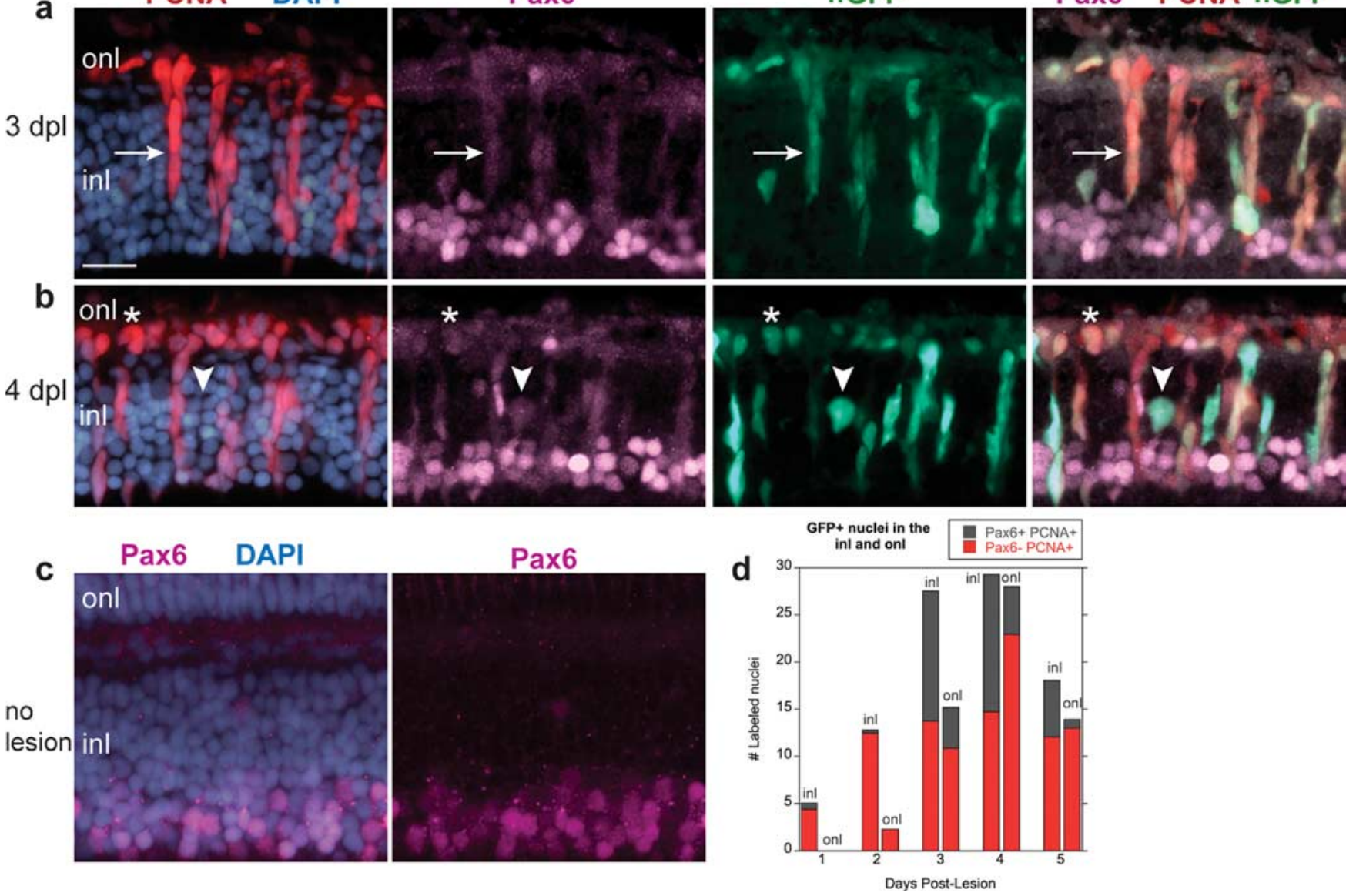

Figure 7. Pax6 is expressed in Müller-derived neuronal progenitors in the regenerating retina. $\boldsymbol{a}-\boldsymbol{c}$, Cryosections of adult zebrafish retinas labeled with Pax6 (magenta), PCNA (red), GFP (green), and DAPI (blue). $\boldsymbol{a}, \boldsymbol{b}$, Light-lesioned retinas from Tg(gfap:nGFP)mizo04 fish. At 3 and $4 \mathrm{dpl}$, almost all GFP ${ }^{+}$nuclei in the inner nuclear layer (inl) are PCNA ${ }^{+}$and weakly Pax6 ${ }^{+}$(arrow). $\boldsymbol{B}, 0$ ne nucleus is GFP ${ }^{+}, \mathrm{Pax}^{+}{ }^{+}$but PCNA ${ }^{-}\left(\boldsymbol{b}\right.$; arrowhead). GFP ${ }^{+}, \mathrm{Pax}^{+}, \mathrm{PCNA}^{+}$cells are also in the outer nuclear layer (onl) $\left({ }^{*}\right) . \boldsymbol{c}$, In unlesioned retinas, expression of Pax6 is primarily restricted to the inner portion of the INL. $\boldsymbol{d}_{\text {, The number of GFP }}{ }^{,}, \mathrm{PCNA}^{+}$nuclei per $100 \mu \mathrm{m}$ linear length of retina in the INL and ONL that are Pax6 ${ }^{+}$(gray) or Pax6 ${ }^{-}$(red) is plotted as a function of days postlesion. Scale bar: (in $\boldsymbol{a}) \boldsymbol{a}-\boldsymbol{c}, 15 \mu \mathrm{m}$.

suggests that $\mathrm{PCNA}^{+}$progenitor cells derived from the injuryinduced proliferating Müller glia initially expressed Pax6 and then downregulated this early retinal progenitor marker while beginning to express the late retinal progenitor marker Crx, which suggests that the injury-induced neuronal progenitors had been specified to the photoreceptor lineage.

\section{Progeny of proliferating Müller glia regenerate cone photoreceptors}

We next asked whether the $\mathrm{GFP}^{+}, \mathrm{Crx}^{+}$cells differentiated into new cone (and rod) photoreceptors to replace those destroyed by the light lesion. To answer this question, we light-lesioned adult $T g(g f a p: n G F P)$ mi2004 fish and labeled retinal sections with the cone-specific monoclonal antibody zpr 1 , which recognizes a cell surface epitope on double cones in zebrafish (Fig. 9d).zpr1 is an early marker of cone differentiation, and immunoreactivity is present within $3 \mathrm{~h}$ of the terminal mitotic division of cone photoreceptors in embryonic zebrafish retina (Larison and Bremiller, 1990). Loss of cone photoreceptors was revealed by disruption of $\mathrm{zpr}^{+}{ }^{+}$cone morphology at $1 \mathrm{dpl}$ and eventually by the absence of zpr1 immunoreactivity at $3 \mathrm{dpl}$ in the lesioned region (Fig. $5 a$ ). Although $\mathrm{GFP}^{+}$nuclei were present in the residual ONL during this period of photoreceptor degeneration (Figs. $6 c, d, 7 a, 8 a$ ), zprl immunostaining first reappeared in the ONL at $4 \mathrm{dpl}$ (Fig. $9 a)$. By $5 \mathrm{dpl}$, the zpr $1^{+}$cells in the ONL were short and squat and resembled "miniature" cones (Fig. 9b). Many of the zpr ${ }^{+}$cells at $5 \mathrm{dpl}$ had the distinctive morphology of immature cone photoreceptors and contained GFP at variable levels of intensity (Fig. $9 b, e)$. By $6 \mathrm{dpl}$, the $\mathrm{zpr} 1^{+}$regenerated cones had further differentiated but they no longer were labeled with the GFP reporter (Fig. 9c). This lineage analysis suggests that regenerated cone photoreceptors derive from injury-induced proliferating Müller glial cells. Rod photoreceptors also regenerated (Fig. $5 g$ ) but with a later time course (Raymond et al., 2006). Although we did not use the GFP lineage-tracing technique to show that these regenerated rods derive from Müller glia, we demonstrated above that they are responsible for the ongoing genesis of rods (Fig. 3). Because cones are generated early in retinogenesis, these data show that the neuronal progenitors produced by Müller glia are not committed to the rod lineage but are multipotent and can revert to an earlier lineage in response to the appropriate microenvironmental signals.

\section{Discussion}

The suggestion that Müller glia are neurogenic is consistent with the established neurogenic role of radial glia in the brain (Anthony et al., 2004; Ever and Gaiano, 2005; Götz and Barde, 2005; Gal et al., 2006; Merkle and Alvarez-Buylla, 2006), but, before the present study, their neurogenic capacity has been observed only in vitro (Das et al., 2006), after retinal injury (Fausett and Gold- 


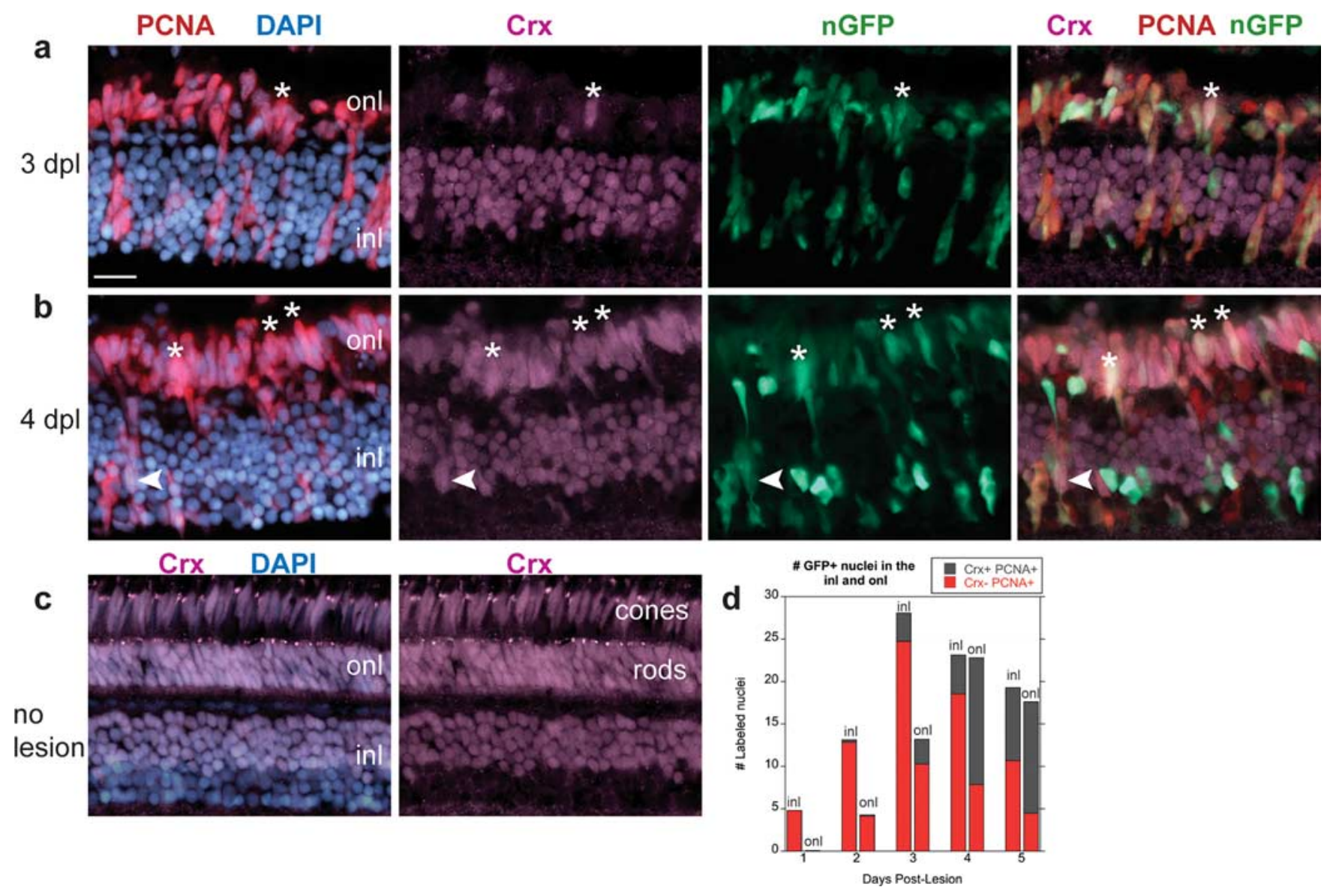

Figure 8. Crx is expressed in Müller-derived photoreceptor progenitors in the regenerating retina. $\boldsymbol{a}-\boldsymbol{c}$, Cryosections of adult zebrafish retinas labeled with Crx (magenta), PCNA (red), GFP

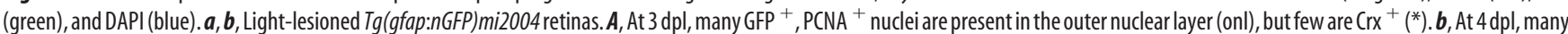
$\mathrm{GFP}^{+}, \mathrm{Crx}^{+}, \mathrm{PCNA}^{+}$nuclei are present in the ONL $\left(^{*}\right)$ and a few in the inner nuclear layer (inl; arrowhead). $c$, An uninjured retina showing the normal expression pattern of $(\mathrm{rx}$ in the outer half of the INL and in the entire ONL, both rod and cone nuclei. $\boldsymbol{d}$, The number of GFP ${ }^{+}, \mathrm{PCNA}^{+}$nuclei per $100 \mu \mathrm{m}$ linear length of retina in the INL and ONL that are $\mathrm{Crx}^{+}$(gray) or Crx ${ }^{-}$(red) is plotted as a function of days postlesion. Scale bar: (in $\boldsymbol{a}) \boldsymbol{a}-\boldsymbol{c}, 15 \mu \mathrm{m}$.

man, 2006; Fimbel et al., 2007), or after intraocular injection of depolarizing neurotoxins or mitogenic growth factors (Fischer and Reh, 2003). One of the most interesting and novel findings from our lineage-tracing study is that Müller glia in the uninjured retina produce the late-stage retinal progenitors of the rod photoreceptor lineage. This observation suggests that the zebrafish retina offers an important model for studying the endogenous, homeostatic neurogenic capacity of retinal Müller glia.

The GFP reporter we used was under the control of glialspecific regulatory elements ( 5 ' and intronic sequences) from the zebrafish gfap genomic locus (Bernardos and Raymond, 2006). Although these gfap transgenic fish lines do not provide a permanent lineage marker as in Cre-LoxP systems, the levels of transgene expression were sufficient that perdurance of the GFP protein after the glial-specific expression was turned off in neuronal progenitors allowed us to trace the $\mathrm{GFP}^{+}$progenitors as they proliferated and differentiated into photoreceptors. Two recent studies examined neurogenesis and neuronal progenitors in injured zebrafish retina with different GFP transgenic lines and alternative injury models. In the first study, regulatory elements of the neuron-specific $\alpha 1$-tubulin gene were identified that activated reporter expression in reactive Müller glia, and a stable transgenic line was created, $\operatorname{Tg}(1016 \alpha 1 T$ :GFP) (Fausett and Goldman, 2006). After stab wound lesions of the retina, GFP expression was seen in proliferating Müller glia and in a few cells that also expressed markers of amacrine and ganglion cells but not in photoreceptors. Because $\alpha 1$-tubulin is a neuron-specific gene widely expressed in neuronal progenitors and immature neurons in the developing brain and retina (Goldman et al., 2001), this analysis could not exclude the possibility that injuryinduced neuronal progenitors not derived from reactive Müller glia might independently have expressed the transgene. The second study followed the regeneration response in retina with three separate transgenic lines, $\operatorname{Tg}(g f a p: E G F P) n t, T g(o l i g 2: E G F P) v u 12$, and $\operatorname{Tg}$ (atoh7:EGFP)nt, in which the transgene was expressed in Müller glia, retinal progenitors, or differentiating retinal ganglion cells, respectively (Fimbel et al., 2007). Intraocular injection of the neurotoxin ouabain destroyed retinal neurons, mainly in the ganglion cell and inner nuclear layers. Müller glia reentered the cell cycle within $1 \mathrm{~d}$, but levels of the GFP reporter in the $\mathrm{Tg}$ (gfap:EGFP) nt transgenic line were insufficient to identify the fate of their differentiated progeny. Instead, the transient expression of these transgenes allowed sequential investigation of the regeneration response: proliferating $\mathrm{GFP}^{+}$retinal progenitors appeared in the $T g$ (olig2:EGFP) vu12 line a few days after ouabain injection, and $\mathrm{GFP}^{+}$regenerating retinal ganglion cells appeared in the $\operatorname{Tg}$ (atoh7:EGFP)nt line over the next several weeks (Fimbel et al., 2007). The spatiotemporal pattern of the regenerative response in the ouabain-damaged zebrafish retina, including the formation of neurogenic clusters that spanned the INL/ONL 
boundary, is consistent with the conclusion that Müller glia are also the source of retinal progenitors in this regeneration paradigm.

Our data provide direct evidence that differentiated Müller glia cells can enter the cell cycle and produce retinal progenitors that differentiate as either rod or cone photoreceptors depending on unidentified extrinsic signals in their microenvironment. We know nothing about the specific extrinsic cues that regulate this photoreceptor lineage specification and that switch progenitor cell fate from rods to cones after injury. We cannot rule out the possibility that a quiescent (nonMüller glia) retinal stem cell population is also present in central retina, and we did observe extremely rare cells that were $\mathrm{GFP}^{-}, \mathrm{Pax}^{+}{ }^{+}, \mathrm{PCNA}^{+}$, but an alternative explanation is that multiple cell divisions might have diluted the GFP reporter to undetectable levels in these cells. Unexpectedly, we also found that the multipotent retinal progenitor marker Pax6 was expressed at low levels in all nondividing, differentiated Müller glia in the zebrafish retina. Expression of Pax6 in radial glia in the brain is associated with the capacity for neurogenesis (Heins et al., 2002; Götz and Barde, 2005) and with maintaining the multipotent state of retinal progenitor cells (Marquardt et al., 2001). One interpretation of our finding that zebrafish Müller glia express Pax6 is that neurogenic capacity is not restricted to a subset of "im-

mature" Müller glia but instead the entire population is competent to generate neuronal progenitors. This interpretation is supported by the observation that injury-induced neurogenic clusters surrounded all Müller glia within the lesion site.

In the neural retina and cerebral cortex, different types of neurons that segregate into distinct laminas are generated sequentially according to a precise timing mechanism thought to involve changes in progenitor cell competence as development proceeds (Livesey and Cepko, 2001; Marquardt and Gruss, 2002; Merkle and Alvarez-Buylla, 2006). Recent models of histogenesis in the retina emphasize the importance of cell-intrinsic regulators and preprogrammed lineages but also acknowledge the influence of extrinsic signals that can modulate cell fate determination (Cayouette et al., 2006). Clonal analysis of neuroepithelial progenitor cells isolated from developing mammalian retina (Cayouette et al., 2003) and cerebral cortex (Shen et al., 2006) and grown in defined medium in cell culture revealed highly regular cell lineages that astonishingly parallel the exact timing and order of histogenesis seen in vivo. These and similar results have reinforced the belief that the capacity of neural progenitors to generate early-born neuronal types is lost at later developmental stages (Götz and Barde, 2005; Merkle and Alvarez-Buylla, 2006).

Our data are at odds with this concept of a unidirectional and progressive pathway of retinal cell fate determination. We show that neurogenic Müller glia in the differentiated zebrafish retina normally produce retinal progenitors that are restricted to the late-born rod photoreceptor lineage, but they are capable of re- sponding to extrinsic signals (loss of cone photoreceptors) to rapidly reset their "lineage clock" and generate early-born neurons (cones) to replace those that were destroyed by exposure to intense light. Although the present study was intentionally limited to analysis of regenerating cones, the robust regenerative ability of the adult teleost fish neural retina is not limited to replacement of photoreceptors but includes restoration of the complete retinal architecture, including the earliest-born neurons, the retinal ganglion cells (Raymond and Hitchcock, 2000; Hitchcock and Raymond, 2004; Fimbel et al., 2007). In all other respects (developmental, functional, and structural), teleost fish have a typical vertebrate retina, but uniquely they retain endogenous retinal stem cells, which we show here are Müller glial cells that have all of the characteristics of genuine adult stem cells: they self-renew, they remain multipotent, and they respond to injury with replacement of damaged tissue. We do not yet understand why Müller glia in other vertebrate retinas are unable to function endogenously as retinal stem cells because they apparently retain a latent potential for neurogenesis.

A model of the teleost Müller glial cell that functions as a radial glial-like progenitor and an injury-induced retinal stem is shown in Figure 10. In the intact, differentiated retina (Fig. 10a), Pax6 ${ }^{+}$ neurogenic Müller glia divide in response to growth-related cues that regulate the addition of new rod photoreceptors in the expanding retina (Otteson and Hitchcock, 2003). We propose that Müller glia divide asymmetrically to produce a retinal progenitor that migrates along the radial process of its sister Müller glia into 

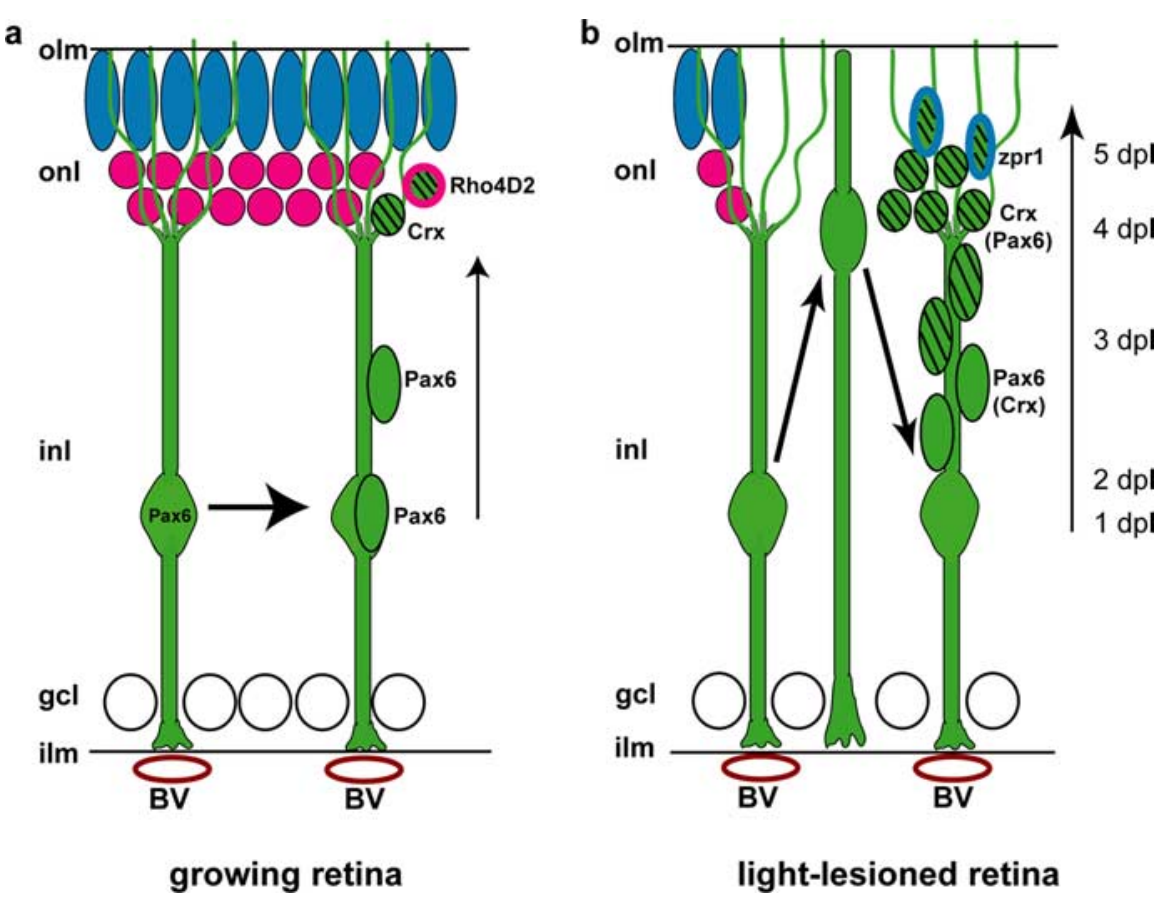

Figure 10. Model of photoreceptor production by Müller glia. $\boldsymbol{a}, \boldsymbol{b}$, Cone photoreceptor nuclei (blue), rod photoreceptor nuclei (magenta), Müller glia and their progeny (green), and blood vessels (BV; red ovals). Additional cells in the inner nuclear layer (inl) have been omitted for simplicity. The radial processes of Müller glia contact blood vessels (BV) at the inner limiting membrane (ilm) and reach the outer limiting membrane (olm). Müller glia nuclei are weakly immunoreactive for Pax6. $\boldsymbol{a}$, Müller glia divide to produce Pax6 ${ }^{+}$neural progenitors in the INL that migrate along the radial glial process to the outer nuclear layer (onl). Pax6 is downregulated in the migrating, proliferating progenitors, and Crx is upregulated. The $\mathrm{Crx}^{+}$progenitors withdraw from the cell cycle and express rod opsin (Rho4D2 immunoreactivity). $\boldsymbol{b}$, After light lesions that destroy photoreceptors, all Müller glia reenter the cell cycle, and their nuclei migrate toward the outer limiting membrane. By $3 \mathrm{dpl}$, many Müller-derived, progenitors in the INL express Pax6, and, by $4 \mathrm{dpl}$, progenitors in the 0NL express Crx. By $5 \mathrm{dpl}$ regenerated, differentiating cones appear in the 0NL and begin to express zpr1, a specific marker of double cones. gcl, Ganglion cell layer.

the ONL (Fig. 10a), similar to neurogenic radial glia in the cortex (Noctor et al., 2001). As they migrate apically along the radial fiber, the retinal progenitors gradually become restricted to the rod photoreceptor lineage, as evidenced by expression of the photoreceptor-specific transcription factor Crx, and eventually they withdraw from the cell cycle and express rod opsin, a marker of differentiated rod photoreceptors. Some direct evidence for asymmetric cell division of Müller glia is the observation of pairs of $\mathrm{GFP}^{+}, \mathrm{BrdU}^{+}$cells, one with the characteristic polygonal nuclear shape of Müller glia and the other a $\mathrm{Crx}^{+}$photoreceptor progenitor. Strong circumstantial evidence for self-renewal is that the linear density of Müller glia remains relatively constant in the uninjured retina despite their continued proliferation, and Müller glial density returns to normal in the repaired retina after a burst of mitotic activity.

In response to apoptosis of photoreceptors (Fig. 10b), nuclei of Müller glia in the region of the lesion migrate apically and reenter the mitotic cycle within a day. This behavior is reminiscent of interkinetic nuclear migration, which is characteristic of neuroepithelial cells and radial glia (Götz and Huttner, 2005). During the regenerative response, reactive Müller glia exhibit other phenotypic changes suggestive of dedifferentiation, including reexpression of BLBP, upregulation of Notch signaling pathways, and redistribution of $N$-cadherin (cdh2) to the basolateral plasma membrane, all of which are characteristics of the multipotent retinal stem cells in the CMZ (Raymond et al., 2006). Together, these findings suggest that the homeostatic and regenerative neurogenic potential of Müller glia is modulated by mi- croenvironmental signals that regulate the cell cycle and the apical-basal polarity of these complex and competent epithelial cells (Kriegstein and Götz, 2003; Malatesta et al., 2003; Götz and Huttner, 2005).

\section{References}

Adolf B, Chapouton P, Lam CS, Topp S, Tannhauser B, Strahle U, Götz M, Bally-Cuif L (2006) Conserved and acquired features of adult neurogenesis in the zebrafish telencephalon. Dev Biol 295:278-293.

Alvarez-Buylla A, Lim DA (2004) For the long run: maintaining germinal niches in the adult brain. Neuron 41:683-686.

Anthony TE, Klein C, Fishell G, Heintz N (2004) Radial glia serve as neuronal progenitors in all regions of the central nervous system. Neuron 41:881-890.

Barthel LK, Raymond PA (1990) Improved method for obtaining 3-micron cryosections for immunocytochemistry. J Histochem Cytochem 38:1383-1388.

Bernardos RL, Raymond PA (2006) GFAP transgenic zebrafish. Gene Expr Patterns 6:1007-1013.

Bernardos RL, Lentz SI, Wolfe MS, Raymond PA (2005) Notch-Delta signaling is required for spatial patterning and Müller glia differentiation in the zebrafish retina. Dev Biol 278:381-395.

Blackshaw S, Harpavat S, Trimarchi J, Cai L, Huang H, Kuo WP, Weber G, Lee K, Fraioli RE, Cho S-H, Yung R, Asch E, Ohno-Machado L, Wong WH, Cepko CL (2004) Genomic analysis of mouse retinal development. PLoS Biol 2:e247.

Braisted JE, Essman TF, Raymond PA (1994) Selective regeneration of photoreceptors in goldfish retina. Development 120:2409-2419.

Bringmann A, Pannicke T, Grosche J, Francke M, Wiedemann P, Skatchkov SN, Osborne NN, Reichenbach A (2006) Müller cells in the healthy and diseased retina. Prog Retin Eye Res 25:397-424.

Cayouette M, Barres BA, Raff M (2003) Importance of intrinsic mechanisms in cell fate decisions in the developing rat retina. Neuron 40:897-904.

Cayouette M, Poggi L, Harris WA (2006) Lineage in the vertebrate retina. Trends Neurosci 29:563-570.

Das AV, Mallya KB, Zhao X, Ahmad F, Bhattacharya S, Thoreson WB, Hegde GV, Ahmad I (2006) Neural stem cell properties of Müller glia in the mammalian retina: regulation by Notch and Wnt signaling. Dev Biol 299:283-302.

Doetsch F (2003) The glial identity of neural stem cells. Nat Neurosci 6:1127-1134.

Ever L, Gaiano N (2005) Radial "glial” progenitors: neurogenesis and signaling. Curr Opin Neurobiol 15:29-33.

Faillace MP, Julian D, Korenbrot JI (2002) Mitotic activation of proliferative cells in the inner nuclear layer of the mature fish retina: regulatory signals and molecular markers. J Comp Neurol 451:127-141.

Fausett BV, Goldman D (2006) A role for $\alpha 1$ tubulin-expressing Müller glia in regeneration of the injured zebrafish retina. J Neurosci 26:6303-6313.

Fimbel SM, Montgomery JE, Burket CT, Hyde DR (2007) Regeneration of inner retinal neurons after intravitreal injection of ouabain in zebrafish. J Neurosci 27:1712-1724.

Fischer AJ (2005) Neural regeneration in the chick retina. Prog Retin Eye Res 24:161-182.

Fischer AJ, Reh TA (2003) Potential of Müller glia to become neurogenic retinal progenitor cells. Glia 43:70-76.

Gal JS, Morozov YM, Ayoub AE, Chatterjee M, Rakic P, Haydar TF (2006) Molecular and morphological heterogeneity of neural precursors in the mouse neocortical proliferative zones. J Neurosci 26:1045-1056.

Goldman D, Hankin M, Li Z, Dai X, Ding J (2001) Transgenic zebrafish for 
studying nervous system development and regeneration. Transgenic Res 10:21-33.

Götz M, Barde YA (2005) Radial glial cells defined and major intermediates between embryonic stem cells and CNS neurons. Neuron 46:369-372.

Götz M, Huttner WB (2005) The cell biology of neurogenesis. Nat Rev Mol Cell Biol 6:777-788.

Heins N, Malatesta P, Cecconi F, Nakafuku M, Tucker KL, Hack MA, Chapouton P, Barde YA, Götz M (2002) Glial cells generate neurons: the role of the transcription factor Pax6. Nat Neurosci 5:308-315.

Hitchcock PF, Raymond PA (2004) The teleost retina as a model for development and regeneration biology. Zebrafish 1:257-271.

Julian D, Ennis K, Korenbrot JI (1998) Birth and fate of proliferative cells in the inner nuclear layer of the mature fish retina. J Comp Neurol 394:271-282.

Kriegstein AR, Götz M (2003) Radial glia diversity: a matter of cell fate. Glia 43:37-43.

Laird DW, Molday RS (1988) Evidence against the role of rhodopsin in rod outer segment binding to RPE cells. Invest Ophthal Vis Sci 29:419-428.

Larison KD, Bremiller R (1990) Early onset of phenotype and cell patterning in the embryonic zebrafish retina. Development 109:567-576.

Lewis GP, Fisher SK (2003) Up-regulation of glial fibrillary acidic protein in response to retinal injury: its potential role in glial remodeling and a comparison to vimentin expression. Int Rev Cytol 230:263-290.

Livesey FJ, Cepko CL (2001) Vertebrate neural cell-fate determination: lessons from the retina. Nat Rev Neurosci 2:109-118.

Malatesta P, Hack MA, Hartfuss E, Kettenmann H, Klinkert W, Kirchhoff F, Götz M (2003) Neuronal or glial progeny: regional differences in radial glia fate. Neuron 37:751-764.

Marquardt T, Gruss P (2002) Generating neuronal diversity in the retina: one for nearly all. Trends Neurosci 25:32-38.

Marquardt T, Ashery-Padan R, Andrejewski N, Scardigli R, Guillemot F, Gruss P (2001) Pax6 is required for the multipotent state of retinal progenitor cells. Cell 105:43-55.

Merkle FT, Alvarez-Buylla A (2006) Neural stem cells in mammalian development. Curr Opin Cell Biol 18:704-709.

Merkle FT, Tramontin AD, Garcia-Verdugo JM, Alvarez-Buylla A (2004) Radial glia give rise to adult neural stem cells in the subventricular zone. Proc Natl Acad Sci USA 101:17528-17532.

Muto A, Orger MB, Wehman AM, Smear MC, Kay JN, Page-McCaw PS, Gahtan E, Xiao T, Nevin LM, Gosse NJ, Staub W, Finger-Baier K, Baier H (2005) Forward genetic analysis of visual behavior in zebrafish. PLoS Genet 1:e66.

Noctor SC, Flint AC, Weissman TA, Dammerman RS, Kriegstein AR (2001) Neurons derived from radial glial cells establish radial units in neocortex. Nature 409:714-720.

Ooto S, Akagi T, Kageyama R, Akita J, Mandai M, Honda Y, Takahashi M (2004) Potential for neural regeneration after neurotoxic injury in the adult mammalian retina. Proc Natl Acad Sci USA 101:13654-13659.

Otteson DC, Hitchcock PF (2003) Stem cells in the teleost retina: persistent neurogenesis and injury-induced regeneration. Vision Res 43:927-936.
Otteson DC, D'Costa AR, Hitchcock PF (2001) Putative stem cells and the lineage of rod photoreceptors in the mature retina of the goldfish. Dev Biol 232:62-76.

Rakic P (1972) Mode of cell migration to the superficial layers of fetal monkey neocortex. J Comp Neurol 145:61-83.

Raymond PA (1985) The unique origin of rod photoreceptors in the teleost retina. Trends Neurosci 8:12-17.

Raymond PA, Hitchcock PF (2000) How the neural retina regenerates. In: Vertebrate eye development (Fini ME, ed), pp 197-218. Berlin: Springer.

Raymond PA, Rivlin PK (1987) Germinal cells in the goldfish retina that produce rod photoreceptors. Dev Biol 122:120-138.

Raymond PA, Reifler MJ, Rivlin PK (1988) Regeneration of goldfish retina: rod precursors are a likely source of regenerated cells. J Neurobiol 19:431-463.

Raymond PA, Barthel LK, Bernardos RL, Perkowski JJ (2006) Molecular characterization of retinal stem cells and their niches in adult zebrafish. BMC Dev Biol 6:36.

Shen Q, Wang Y, Dimos JT, Fasano CA, Phoenix TN, Lemischka IR, Ivanova NB, Stifani S, Morrisey EE, Temple S (2006) The timing of cortical neurogenesis is encoded within lineages of individual progenitor cells. Nat Neurosci 9:743-751.

Shen YC, Raymond PA (2004) Zebrafish cone-rod (crx) homeobox gene promotes retinogenesis. Dev Biol 269:237-251.

Shi SR, Chaiwun B, Young L, Imam A, Cote RJ, Taylor CR (1994) Antigen retrieval using $\mathrm{pH} 3.5$ glycine- $\mathrm{HCl}$ buffer or urea solution for immunohistochemical localization of Ki-67. Biotech Histochem 69:213-215.

Turner DL, Cepko CL (1987) A common progenitor for neurons and glia persists in rat retina late in development. Nature 328:131-136.

Vihtelic TS, Hyde DR (2000) Light-induced rod and cone cell death and regeneration in the adult albino zebrafish (Danio rerio) retina. J Neurobiol 44:289-307.

Vihtelic TS, Soverly JE, Kassen SC, Hyde DR (2006) Retinal regional differences in photoreceptor cell death and regeneration in light-lesioned albino zebrafish. Exp Eye Res 82:558-575.

Westerfield M (2000) The zebrafish book. A guide for the laboratory use of zebrafish (Danio rerio), Ed 4. Eugene, OR: University of Oregon.

Wetts R, Fraser SE (1988) Multipotent precursors can give rise to all major cell types of the frog retina. Science 239:1142-1145.

Wu DM, Schneiderman T, Burgett J, Gokhale P, Barthel L, Raymond PA (2001) Cones regenerate from retinal stem cells sequestered in the inner nuclear layer of adult goldfish retina. Invest Ophthal Vis Sci 42:2115-2124.

Yurco P, Cameron DA (2005) Responses of Müller glia to retinal injury in adult zebrafish. Vision Res 45:991-1002.

Zupanc GK, Clint SC (2003) Potential role of radial glia in adult neurogenesis of teleost fish. Glia 43:77-86.

Zupanc GK, Hinsch K, Gage FH (2005) Proliferation, migration, neuronal differentiation, and long-term survival of new cells in the adult zebrafish brain. J Comp Neurol 488:290-319. 